TRANSACTIONS OF THE

AMERICAN MATHEMATICAL SOCIETY

Volume 354, Number 11, Pages 4639-4679

S 0002-9947(02)03059-3

Article electronically published on July 2, 2002

\title{
GAUGEABILITY AND CONDITIONAL GAUGEABILITY
}

\author{
ZHEN-QING CHEN
}

\begin{abstract}
New Kato classes are introduced for general transient Borel right processes, for which gauge and conditional gauge theorems hold. These new classes are the genuine extensions of the Green-tight measures in the classical Brownian motion case. However, the main focus of this paper is on establishing various equivalent conditions and consequences of gaugeability and conditional gaugeability. We show that gaugeability, conditional gaugeability and the subcriticality for the associated Schrödinger operators are equivalent for transient Borel right processes with strong duals. Analytic characterizations of gaugeability and conditional gaugeability are given for general symmetric Markov processes. These analytic characterizations are very useful in determining whether a process perturbed by a potential is gaugeable or conditionally gaugeable in concrete cases. Connections with the positivity of the spectral radii of the associated Schrödinger operators are also established.
\end{abstract}

\section{INTRODUCTION}

Given a strong Markov process $X$ and a potential $q$, the conditional expectation $u(x, y)$ of the Feynman-Kac transform of $X$ by $q$ is called the conditional gauge function. (The precise definition will be given later.) The conditional gauge function $u$ is important in studying the potential theory of the Schrödinger-type operator $\mathcal{L}+q$, as it is the ratio of the Green function of $\mathcal{L}+q$ and that of $\mathcal{L}$ (see Lemma 3.5 below), where $\mathcal{L}$ is the infinitesimal generator of $X$. The conditional gauge theorem says that under suitable conditions for $X$ and $q$, either $u$ is identically infinite or $u$ is bounded between two positive numbers. The conditional gauge theorem was first proved for Brownian motions by Falkner, Zhao, Chung and Cranston, Fabes and Zhao (see Chung and Zhao [13] for the history). It was recently established by Chen and Song in 7 for symmetric stable processes. Very recently general gauge and conditional gauge theorems have been established by Chen and Song in [8] and 9] for a large class of strong Markov processes which may not be symmetric or have continuous sample paths and for a large class of potentials. Conditional gauge theorems are much deeper than gauge theorems, which state that under suitable conditions for $X$ and $q$, the expected value of the Feynman-Kac transform of $X$ by $q$ as a function of the starting point of $X$ is either bounded or identically infinite.

Received by the editors August 12, 2001 and, in revised form, February 7, 2002.

2000 Mathematics Subject Classification. Primary 60J45, 60J57; Secondary 35J10, 35S05, 47J20, 60J35.

Key words and phrases. Green function, $h$-transform, conditional Markov process, lifetime, time change, Kato class, Feynman-Kac transform, Schrödinger semigroup, Stieltjes exponential, non-local perturbation, spectral radius, gauge theorem, conditional gauge theorem, super gauge theorem, super conditional gauge theorem, subcriticality, bilinear form.

The research of this author is supported in part by NSF Grant DMS-0071486. 
The gauge theorem for Brownian motion was first proved by Chung and Rao in [11] for bounded $q$ and later was generalized to more general $q$ by various authors. For the history of this theorem, we refer the reader to the recent book by Chung and Zhao [12]. Gauge theorems have been proved for quite general Markov processes by Chung and Rao 12 and by Sturm 38 under some variant assumptions and settings than those by Chen and Song in [8] and [9]. The approach to the general gauge theorem established in [8] and [9] is influenced by those in Chung and Rao [12 and in Section 5.6 of Chung and Zhao 13 . But it is modified and extended in some directions, and is tailored so that it can be applied to conditional processes to yield the conditional gauge theorem for a large class of Markov processes.

The Kato classes introduced in [8] and 9] are for potentials given by functions $q$ and do not cover potentials given by measures $\mu$ that are not absolutely continuous with respect to the reference measure. For example, it does not include the case where the potential is the surface measure of a sphere when the process is a Brownian motion in $\mathbf{R}^{n}$.

In this paper we introduce new Kato classes under which the general gauge and conditional gauge theorems hold and they include singular measures. In fact, we show that these are the genuine extensions of the Green-tight measures introduced by Zhao 43. for Brownian motion in $\mathbf{R}^{n}$. However, the main focus of this paper is to establish various equivalent conditions and consequences of gaugeability and conditional gaugeability, aimed at addressing the question when the gauge or conditional gauge function is bounded. We show that gaugeability, conditional gaugeability and subcriticality for the associated Schrödinger operators are equivalent for transient Borel right processes with strong duals. Analytic characterizations of gaugeability and conditional gaugeability are given for general symmetric Markov processes, extending the recent results of Takeda [40]. These analytic characterizations are very useful in determining whether $(X, \mu)$ is gaugeable or conditionally gaugeable in concrete cases. To get these analytic characterizations, we first prove a result about the independence of the $L^{p}$-spectral radius, by applying a conditional gauge theorem. Connections with the positivity of the spectral radii of the associated Schrödinger operators are also established. Furthermore, general gauge and conditional gauge theorems for non-local perturbed Schrödinger operators (that is, Schrödinger operators obtained through Feynman-Kac transforms by discontinuous additive functionals) are also established in this paper together with their various equivalent conditions. To ensure a broad and easy applicability of the results in this paper, we have strived to impose as few constraints on the processes and their potentials as possible. We point out that, in addition to their validity in the most general settings so far, some results and approaches in this paper are new even in the classical case when $X$ is a Brownian motion in $\mathbf{R}^{n}$ or in a domain of $\mathbf{R}^{n}$.

The conditional gauge theorem and its equivalence results for discontinuous additive functionals will be applied in a forthcoming paper [10] jointly with Song to study discontinuous Markov processes under pure jump Girsanov transforms and their Green functions estimates.

The rest of the paper is organized as follows. In section 2, we introduce new Kato classes for general transient Borel right processes having Green functions and establish a gauge theorem for potentials from these new classes. Connections with classical Kato classes, with classes introduced by Chen and Song in [8] and [9], and with $G$-Kato measures introduced by Amor and Hansen [2] are discussed in detail. Section 3 introduces new Kato classes for conditional processes and proves 
the conditional gauge theorem under the strong duality assumption. Several equivalent conditions for gaugeability and conditional gaugeability, including the condition of positive spectral radii and the condition of subcriticality for the associated Schrödinger operators, are given in sections 2 and 3 respectively. General gauge and conditional gauge theorems for discontinuous additive functionals under new Kato classes and their equivalent conditions are also presented, as well as super gauge and super conditional gauge theorems. In section 4, we prove the independence of the $L^{p}$-spectral radius for Borel right processes with strong duals. In section 5 , we give analytic characterizations when $(X, \mu)$ is gaugeable or conditionally gaugeable under the assumption that the process $X$ is symmetric.

To keep the exposition as transparent as possible, in sections 2 and 3 we first present and prove results for Feynman-Kac transformations by continuous additive functionals. We then present and prove results for Feynman-Kac transformations given by more general discontinuous additive functionals. The latter is done under the context that the underlying process is a Borel standard process, instead of being a Borel right process.

In this paper, we denote ":=" to mean "is defined to be". For functions $f$ and $g$, notation " $f \approx g$ " means that there exist constants $c_{2}>c_{1}>0$ such that $c_{1} g \leq f \leq c_{2} g$. For two real numbers $a$ and $b, a \wedge b:=\min \{a, b\}$. For a set $K$ in a space $E$, we use $K^{c}$ to denote its complement in $E$, that is, $K^{c}:=E \backslash K$. For a function $f$ defined on $E,\|f\|_{\infty}:=\sup _{x \in E}|f(x)|$.

\section{Kato Classes AND GaUgeability}

Let $E$ be a Lusin space (i.e., a space that is homeomorphic to a Borel subset of a compact metric space) and let $\mathcal{B}(E)$ be the Borel $\sigma$-algebra on $E$. Let $X=$ $\left(\Omega, \mathcal{M}, \mathcal{M}_{t}, X_{t}, \mathbf{P}_{x}, x \in E\right)$ be a Borel right process on $E$ having left limits on $(0, \zeta)$ that is transient in the sense of Getoor [23. Here, a Borel right process on a Lusin space $E$ is a right continuous, strong Markov process with no branching points and with a Borel measurable resolvent. The shift operators $\theta_{t}, t \geq 0$, satisfy $X_{s} \circ \theta_{t}=X_{s+t}$ identically for $s, t \geq 0$. Adjoined to the state space $E$ is an isolated point $\partial \notin E$; the process $X$ retires to $\partial$ at its "lifetime" $\zeta:=\inf \left\{t \geq 0: X_{t}=\partial\right\}$. Denote $E \cup\{\partial\}$ by $E_{\partial}$. Let $m$ be an excessive measure of $X$ with $\operatorname{supp}[m]=E$; that is, $m$ is a $\sigma$-finite Borel measure on $\mathcal{B}(E)$ such that $m P_{t} \leq m$ for all $t>0$. Here $m P_{t}$ denotes the measure $\mu$ defined by $\int_{E} f(x) \mu(d x)=\int_{E} P_{t} f(x) m(d x)$ for any Borel function $f \geq 0$ on $E$. Since $X$ is a Borel right process, we have $\lim _{t \rightarrow 0} m P_{t}=m$ setwise. Throughout this paper, the process $X$ is assumed to be $m$-irreducible in the sense that if a measurable set $A$ has positive $m$-measure, then $\mathbf{P}_{x}\left[T_{A}<\infty\right]>0$ for all $x \in E$, where $T_{A}=\inf \left\{t>0, X_{t} \in A\right\}$ is the first hitting time of $A$.

The transition operators $P_{t}, t \geq 0$, are defined by

$$
P_{t} f(x):=\mathbf{E}_{x}\left[f\left(X_{t}\right)\right]=\mathbf{E}_{x}\left[f\left(X_{t}\right) ; t<\zeta\right] .
$$

(Here and in the sequel, unless mentioned otherwise, we use the convention that a function defined on $E$ takes the value 0 at the cemetery point $\partial$.)

We assume that there is a Borel function $G(x, y)$ on $E \times E$ such that

$$
\mathbf{E}_{x}\left[\int_{0}^{\infty} f\left(X_{s}\right) d s\right]=\int_{E} G(x, y) f(y) m(d y)
$$

for all measurable $f \geq 0$. $G(x, y)$ is called the Green function of $X$. 
A set $B$ is said to be $m$-polar if $\mathbf{P}_{m}\left(\sigma_{B}<\infty\right)=0$, where $\sigma_{B}:=\inf \left\{t>0: X_{t} \in\right.$ $B\}$, and $m$-semipolar if

$$
\mathbf{P}_{m}\left(X_{t} \in B \text { for uncountably many } t\right)=0 .
$$

We call a positive measure $\mu$ on $E$ a smooth measure of $X$ if there is a positive continuous additive functional (PCAF in abbreviation) $A$ of $X$ such that

$$
\int_{E} f(x) \mu(d x)=\uparrow \lim _{t \downarrow 0} \mathbf{E}_{m}\left[\frac{1}{t} \int_{0}^{t} f\left(X_{s}\right) d A_{s}\right]
$$

for any Borel $f \geq 0$. Here $\uparrow \lim _{t \downarrow 0}$ means the quantity is increasing as $t \downarrow 0$. The measure $\mu$ is called the Revuz measure of $A$. Throughout this paper, all additive functionals should be understood in the strict sense, i.e., in the sense of [4. Recall that since $X$ is assumed to have a Green function, any $m$-polar set is polar. When $X$ admits a strong dual, a PCAF having bounded 1-potential in the sense of [20] with an exceptional set can be uniquely refined into a PCAF in the strict sense. This can be proved by using the same argument as that in the proof of Theorem 5.1.6 of Fukushima, Oshima and Takeda [22. Hence in this context, Fitzsimmons and Getoor 20] gives a one-to-one correspondence between smooth measures having bounded 1-potential and PCAF in the strict sense having bounded 1-potential. Here we just mention that such a smooth measure $\mu$ is $\sigma$-finite, charges no $m$-semipolar sets and uniquely determines $A$. It is shown in Proposition 3.5 of Getoor [24] that a positive measure $\mu$ charging no $m$-semipolar sets with $\left\|G_{\alpha} \mu\right\|_{\infty}<\infty$ for some $\alpha>0$ is a smooth measure. When $X$ is in strong duality (with respect to $m$ ) with a second Borel right process $\widehat{X}$, and the Silverstein's sector condition is satisfied by $X$ and $m$, and $\widehat{G}_{\alpha_{0}} 1$ is lower semi-continuous for some $\alpha_{0}>0$, then $\left\|G_{\alpha} \mu\right\|_{\infty}<\infty$ implies $\mu$ charges no $m$-polar sets (cf. [20]), and therefore by Silverstein 34] $\mu$ charges no $m$-semipolar sets. So $\mu$ is a smooth measure in this case.

For a positive smooth measure $\mu$, we define its potential $U \mu$ by

$$
U \mu(x):=\mathbf{E}_{x}\left[A_{\infty}\right],
$$

where $A$ is the PCAF with Revuz measure $\mu$. Note that if $K$ is a Borel subset of $E$, then $1_{K} \mu$ is the Revuz measure for PCAF $t \mapsto \int_{0}^{t} 1_{K}\left(X_{s}\right) d A_{s}$. If $\mu(d x)=$ $f(x) m(d x)$, clearly

$$
U \mu(x)=G f(x):=\int_{E} G(x, y) f(y) m(d y) .
$$

If some additional regularity condition is satisfied by process $X$ and its Green function $G(x, y)$, for example, when $X$ has a dual Borel Markov process $\widehat{X}$ or when the Green function $G(x, y)$ of $G$ satisfies certain regularity conditions as specified in Meyer [29], then for smooth measure $\mu$,

$$
U \mu(x)=\int_{E} G(x, y) \mu(d y) .
$$

The latter will be abbreviated as $G \mu(x)$.

2.1. New Kato Classes. The following definitions are from Chen and Song [8] and [9], and the classes in (2) and (3) are now denoted as $\widetilde{\mathbf{K}}_{\infty}(X)$ and $\widetilde{\mathbf{K}}_{1}(X)$ respectively. For a signed measure $\mu$, we use $\mu^{+}$and $\mu^{-}$to denote its positive and negative parts, respectively. 
Definition 2.1. Suppose that $\mu$ is a signed smooth measure whose associated continuous additive functional of $X$ is $A$. Let $A^{+}$and $A^{-}$be the PCAFs of $X$ with Revuz measures $\mu^{+}$and $\mu^{-}$, respectively. Let $|A|=A^{+}+A^{-}$and $|\mu|=\mu^{+}+\mu^{-}$.

(1) Measure $\mu$ is said to be in the Kato class of $X, \mathbf{K}(X)$ in abbreviation, if

$$
\lim _{t \rightarrow 0} \sup _{x \in E} \mathbf{E}_{x}\left[|A|_{t}\right]=0 .
$$

(2) Measure $\mu$ is said to be in the class $\widetilde{\mathbf{K}}_{\infty}(X)$ if for any $\varepsilon>0$, there is a Borel set $K=K(\varepsilon)$ of finite $m$-measure and a constant $\delta=\delta(\varepsilon)>0$ such that

$$
\left\|U\left(1_{K^{c}}|\mu|\right)\right\|_{\infty}<\varepsilon \text { and }\left\|U\left(1_{B}|\mu|\right)\right\|_{\infty}<\varepsilon
$$

for all measurable sets $B \subset K$ with $m(B)<\delta$.

(3) Measure $\mu$ is said to be in the class $\widetilde{\mathbf{K}}_{1}(X)$ if there is a Borel set $K$ of finite $m$-measure and a constant $\delta>0$ such that

$$
\widetilde{\beta}_{1}(\mu):=\sup _{B \subset K: m(B)<\delta}\left\|U\left(1_{K^{c} \cup B}|\mu|\right)\right\|_{\infty}<1 .
$$

In Chen and Song $[\underline{8}$, the gauge theorem was proved for measures $\mu=q(x) m(d x)$ in $\widetilde{\mathbf{K}}_{1}(X)$. Clearly, $\widetilde{\mathbf{K}}_{\infty}(X) \subset \widetilde{\mathbf{K}}_{1}(X)$. It is easy to see that if a measure $\mu$ satisfies condition (2.3), then $\mu(d x)=q(x) m(d x)$ for some $q$. More precisely, there is a function $q$ such that $U \mu=G q$. Here $G q(x):=\int_{E} G(x, y) q(y) m(d y)$. So $\widetilde{\mathbf{K}}_{\infty}(X)$ excludes interesting cases of measures that are not absolutely continuous with respect to $m$. We now introduce two new Kato classes of measures $\mathbf{K}_{\infty}(X)$ and $\mathbf{K}_{1}(X)$, which are strict extensions of $\widetilde{\mathbf{K}}_{\infty}(X)$ and $\widetilde{\mathbf{K}}_{1}(X)$ respectively, and under which the gauge theorem still holds.

Definition 2.2. Suppose that $\mu$ is a smooth measure on $E$.

(1) $\mu$ is said to be in the class $\mathbf{K}_{\infty}(X)$ if for any $\varepsilon>0$, there is a Borel set $K=K(\varepsilon)$ of finite $|\mu|$-measure and a constant $\delta=\delta(\varepsilon)>0$ such that

$$
\left\|U\left(1_{K^{c}}|\mu|\right)\right\|_{\infty}<\varepsilon \text { and }\left\|U\left(1_{B}|\mu|\right)\right\|_{\infty}<\varepsilon
$$

for all measurable sets $B \subset K$ with $|\mu|(B)<\delta$.

(2) $\mu$ is said to be in the class $\mathbf{K}_{1}(X)$ if there is a Borel set $K$ of finite $|\mu|$-measure and a constant $\delta>0$ such that

$$
\beta_{1}(\mu):=\sup _{B \subset K:|\mu|(B)<\delta}\left\|U\left(1_{K^{c} \cup B}|\mu|\right)\right\|_{\infty}<1 .
$$

(3) A function $q$ is said to be in a class $\mathbf{K}(X), \mathbf{K}_{\infty}(X)$, or $\mathbf{K}_{1}(X)$, if $\mu(d x):=$ $q(x) m(d x)$ is in the corresponding spaces.

Clearly $\mathbf{K}_{\infty}(X) \subset \mathbf{K}_{1}(X)$. Note that $\mu$ is $\sigma$-finite; so from measure theory we know that the set $K$ in the above definitions can be taken to be a closed set or an open set (see, e.g., Exercise 18 on page 31 of Folland [21]). The introduction of new classes $\mathbf{K}_{\infty}(X)$ and $\mathbf{K}_{1}(X)$ allows us to include measures that are not absolutely continuous with respect to the measure $m$, while still preserving the validity of the gauge theorem. Take $X$ to be a Brownian motion in $\mathbf{R}^{n}$ with $n \geq 3$, for example, and take $\mu$ to be the surface measure of the unit sphere in $\mathbf{R}^{n}$. Then it is easy to check directly that $\mu \in \mathbf{K}_{\infty}(X)$. In fact, we have the following.

Theorem 2.1. Let $X$ be a symmetric $\alpha$-stable process in $\mathbf{R}^{n}$ with $0<\alpha \leq 2$ and $n>\alpha$; so the Green function of $X$ is $G(x, y)=c(n, \alpha)|x-y|^{\alpha-n}$. 
(1) A signed measure $\mu$ is in $\mathbf{K}(X)$ if and only if it is a Kato measure in the classical sense; that is,

$$
\lim _{r \rightarrow 0} \sup _{x \in \mathbf{R}^{n}} \int_{B(x, r)}|x-y|^{\alpha-n}|\mu|(d y)=0 .
$$

Here $B(x, r)$ denotes the ball in $\mathbf{R}^{n}$ centered at $x$ with radius $r$.

(2) A finite signed measure $\mu$ is in $\mathbf{K}_{\infty}(X)$ if and only if it satisfies (2.7).

(3) A signed measure $\mu$ is in $\mathbf{K}_{\infty}(X)$ if and only if it is Green-tight in the sense of Zhao [3]; that is, both (2.7) and the equation

$$
\lim _{R \rightarrow \infty} \sup _{x \in \mathbf{R}^{n}} \int_{B(0, R)^{c}}|x-y|^{\alpha-n}|\mu|(d y)=0
$$

are satisfied.

Before proving this theorem, we first establish the following.

Proposition 2.2. For $\mu \in \mathbf{K}_{1}(X), U|\mu|$ is bounded.

Proof. Without loss of generality, we assume that $\mu \in \mathbf{K}_{1}(X)$ is a nonnegative measure. Let $K$ be the Borel set of finite $\mu$-measure and $\delta$ be the constant as in Definition 2.2 for $\mu$. Set $K$ can have at most finitely many atoms $\left\{w_{1}, \cdots, w_{k}\right\}$ of size larger than $\delta / 2$ for measure $\mu$. The remaining $K \backslash\left\{x_{1}, \cdots, w_{k}\right\}$ can be covered by finitely many Borel subsets $\left\{B_{i}, 1 \leq i \leq l\right\}$ with $\mu\left(B_{i}\right)<\delta$. Since $X$ is transient and $\mu$ is a smooth measure that does not charge on polar sets, $m\left(\left\{w_{i}\right\}\right)>0$ and therefore by Getoor [23] $\left\|U \delta_{\left\{w_{i}\right\}}\right\|_{\infty}<\infty$ for $1 \leq i \leq k$, where $\delta_{\left\{w_{i}\right\}}$ denotes the measure with unit mass concentrated at $w_{i}$. Hence

$$
\|U \mu\|_{\infty} \leq\left\|U\left(1_{K^{c}} \mu\right)\right\|_{\infty}+\sum_{i=1}^{k}\left\|U\left(1_{\left\{w_{i}\right\}} \mu\right)\right\|_{\infty}+\sum_{i=1}^{l}\left\|U\left(1_{B_{i}} \mu\right)\right\|_{\infty}<\infty .
$$

Proof of Theorem [2.1. We only need to prove this theorem for positive measure $\mu$. Note that the symmetric stable process $X$ is a Hunt process and so the Revuz formula 2.2 holds.

(1) This was proved as Theorem 1 in Zhao 42 .

(2) If $\mu \in \mathbf{K}_{\infty}(X), G \mu$ is bounded by Proposition 2.2. Since

$$
c(n, \alpha) r^{\alpha-n} \mu(B(x, r)) \leq \int_{B(x, r)} G(x, y) \mu(d y) \leq\|G \mu\|_{\infty},
$$

one has

$$
\mu(B(x, r)) \leq c(n, \alpha)^{-1}\|G \mu\|_{\infty} r^{n-\alpha},
$$

which converges uniformly in $x \in \mathbf{R}^{n}$ to 0 as $r \rightarrow 0$. It follows immediately from the definition for $\mathbf{K}_{\infty}(X)$ that (2.7) holds for $\mu$. Conversely, suppose that $\mu$ is a positive finite measure satisfying (2.7). Note that for any measurable set $A$,

$$
\begin{aligned}
\int_{A} G(x, y) \mu(d y) \leq & c(n, \alpha) \int_{B(x, r)}|x-y|^{\alpha-n} \mu(d y) \\
& \quad+c(n, \alpha) \int_{A \cap B(x, r)^{c}}|x-y|^{\alpha-n} \mu(d y) \\
\leq & c(n, \alpha) \sup _{x \in \mathbf{R}^{n}} \int_{B(x, r)}|x-y|^{\alpha-n} \mu(d y)+c(n, \alpha) r^{\alpha-n} \mu(A) .
\end{aligned}
$$


Since $\mu$ is a finite measure satisfying (2.7), we see that for any given $\varepsilon>0$ there is a ball $K=B(0, R)$ such that

$$
\sup _{x \in \mathbf{R}^{n}} \int_{K^{c}} G(x, y) \mu(d y)<\varepsilon .
$$

This is achieved by first selecting $r$ small and then taking $R$ sufficiently large for $A=B(0, R)^{c}$ in (2.10). It also follows from (2.10) that there is $\delta=\delta(\varepsilon)>0$ such that for any Borel subset $B \subset K$ with $\mu(B)<\delta$,

$$
\int_{B} G(x, y) \mu(d y)<\varepsilon \quad \text { for all } x \in \mathbf{R}^{n} .
$$

This proves that $\mu \in \mathbf{K}_{\infty}(X)$.

(3) Suppose that $\mu$ is a positive measure in $\mathbf{K}_{\infty}(X)$. We already see from the proof above that $\mu$ satisfies (2.7). For $\varepsilon>0$, let $K=K(\varepsilon)$ and $\delta=\delta(\varepsilon)$ be the Borel set of finite $\mu$-mass and the constant in the definition of $\mu \in \mathbf{K}_{\infty}(X)$. Since $\mu\left((0, R)^{c} \cap K\right) \rightarrow 0$ as $R \rightarrow \infty$, we have

$$
\sup _{x \in \mathbf{R}^{n}} \int_{B(0, R)^{c} \cap K} G(x, y) \mu(d y)<\varepsilon
$$

when $R$ is large enough. Therefore,

$$
\begin{aligned}
& \sup _{x \in \mathbf{R}^{n}} \int_{B(0, R)^{c}} G(x, y) \mu(d y) \\
& \quad \leq \sup _{x \in \mathbf{R}^{n}}\left(\int_{K^{c}} G(x, y) \mu(d y)+\int_{B(0, R)^{c} \cap K} G(x, y) \mu(d y)\right) \leq 2 \varepsilon,
\end{aligned}
$$

and so $\mu$ is Green-tight.

Now suppose $\mu$ is a positive measure that is Green-tight. For any $\varepsilon>0$, let $R>0$ be such that

$$
\sup _{x \in \mathbf{R}^{n}} \int_{B(0, R)^{c}} G(x, y) \mu(d y)<\varepsilon .
$$

It follows from (2.7) that $\mu(B(0, R))<\infty$ and by (2.10),

$$
\lim _{\delta \rightarrow 0} \sup _{x \in \mathbf{R}^{n}} \sup _{B \subset B(0, R): \mu(B)<\delta} \int_{B} G(x, y) \mu(d y)=0 .
$$

Thus $\mu$ is in $\mathbf{K}_{\infty}(X)$.

Remark 2.1. Theorem 2.1 says that the new classes $\mathbf{K}_{\infty}(X)$ and $\mathbf{K}_{1}(X)$ are genuine extensions of Green-tight measures to the general Markov process $X$.

For positive smooth measure $\mu$ and $\alpha>0$, its $\alpha$-potential $U_{\alpha} \mu$ is defined as

$$
U_{\alpha} \mu(x):=\mathbf{E}_{x}\left[\int_{0}^{\infty} e^{-\alpha t} d A_{t}\right], \quad x \in E,
$$

where $A$ is the PCAF of $X$ having Revuz measure $\mu$.

Proposition 2.3. Assume $\mu$ is a positive measure.

(1) If $\mu \in \mathbf{K}_{\infty}(X)$, then $\lim _{\alpha \rightarrow \infty}\left\|U_{\alpha} \mu\right\|_{\infty}=0$. Therefore we have $\mathbf{K}_{\infty}(X) \subset$ $\mathbf{K}(X)$.

(2) If $\mu \in \mathbf{K}_{1}(X)$, then $\lim _{\alpha \rightarrow \infty}\left\|U_{\alpha} \mu\right\|_{\infty} \leq \beta_{1}(\mu)<1$. 
Proof. (1) Let $\mu$ be a positive measure in $\mathbf{K}_{\infty}(X)$ and $A$ its associated PCAF of $X$. For any given $\varepsilon>0$, let $K=K(\varepsilon)$ be the closed set of finite $\mu$-measure and $\delta=\delta(\varepsilon)$ the constant in Definition 2.2(2). Since $\alpha U_{\alpha} U \mu$ increases to $U \mu$ on $E$ as $\alpha \uparrow \infty$, by taking $\alpha$ large enough, the set $B=\left\{x \in K: U \mu(x)-\alpha U_{\alpha} U(x) \mu>\varepsilon\right\}$ has $\mu$ measure less than $\delta$. Since $U_{\alpha}=U-\alpha U_{\alpha} U$, the set $K \backslash B=\left\{x \in K: U_{\alpha} \mu(x) \leq \varepsilon\right\}$ is finely closed and therefore $X_{\sigma_{K \backslash B}} \in K \backslash B$, where $\sigma_{K \backslash B}$ is the first hitting time of $K \backslash B$ by $X$. Thus by the strong Markov property,

$$
\begin{aligned}
U_{\alpha} \mu(x) & =U\left(1_{K^{c}} \mu\right)(x)+U\left(1_{B} \mu\right)(x)+U_{\alpha}\left(1_{K \backslash B} \mu\right)(x) \\
& \leq \varepsilon+\varepsilon+\mathbf{E}_{x}\left[U_{\alpha}\left(1_{K \backslash B} \mu\right)\left(X_{\sigma_{K \backslash B}}\right)\right] \\
& \leq 3 \varepsilon .
\end{aligned}
$$

So we have $\left\|U_{\alpha} \mu\right\|_{\infty} \rightarrow 0$ as $\alpha \uparrow \infty$, which is equivalent to $\sup _{x \in E} \mathbf{E}_{x}\left[A_{t}\right] \rightarrow 0$ as $t \downarrow 0$. Hence $\mu \in \mathbf{K}(X)$.

(2) The same estimate above shows that for $\mu \in \mathbf{K}_{1}(X), \lim _{\alpha \rightarrow \infty}\left\|U_{\alpha} \mu\right\|_{\infty} \leq$ $\beta_{1}(\mu)$.

In [2], Amor and Hansen introduced a class of positive measures which they call $G$-Kato measures. In the following we show that the $G$-Kato class measures are exactly the $\mathbf{K}_{\infty}(X)$-measures.

Proposition 2.4. Let $\mu$ be a positive smooth measure on $E$.

(1) $\mu$ is in $\mathbf{K}_{\infty}(X)$ if and only if it is a $G$-Kato measure; that is, $U \mu(x)$ is bounded and for every sequence of Borel sets $F_{n}$ that increases to $E, U\left(1_{F_{n}} \mu\right)$ increases to $U \mu$ uniformly on $E$.

(2) $\mu$ is in $\mathbf{K}_{1}(X)$ if and only if $U \mu$ is bounded and there is a constant $\beta \in(0,1)$ such that for every decreasing sequence $\left\{D_{n}, n \geq 1\right\}$ of Borel sets with empty intersection, $\lim _{n \rightarrow \infty}\left\|U\left(1_{D_{n}} \mu\right)\right\|_{\infty} \leq \beta$.

Proof. (1) Suppose that $\mu$ is a positive measure in $\mathbf{K}_{\infty}(X)$. By Proposition 2.2 $U \mu$ is bounded. Let $F_{n}$ be a sequence of Borel sets that increases to $E$. For any given $\varepsilon>0$, let $K=K(\varepsilon)$ be the Borel set of finite $\mu$-measure and $\delta=\delta(\varepsilon)>0$ the constant in Definition 2.2 of $\mathbf{K}_{\infty}(X)$ for $\mu$. As $\lim _{n \rightarrow \infty} \mu\left(K \backslash F_{n}\right)=0$, there is an integer $N>0$ such that $\mu\left(K \backslash F_{n}\right)<\delta$ for $n>N$. Hence for $n>N$,

$$
\left\|U \mu-U\left(1_{F_{n}} \mu\right)\right\|_{\infty} \leq\left\|U\left(1_{K^{c}} \mu\right)\right\|_{\infty}+\left\|U\left(1_{K \backslash F_{n}} \mu\right)\right\|_{\infty}<2 \varepsilon .
$$

This says that $U\left(1_{F_{n}} \mu\right)$ increases to $U \mu$ uniformly on $E$ and so $\mu$ is a $G$-Kato measure.

Conversely, suppose $\mu$ is a positive $G$-Kato class smooth measure. Since $\mu$ is $\sigma$-finite and since one can exhaust $E$ by an increasing sequence of Borel sets of finite $\mu$-measures, for any give $\varepsilon>0$, there is a Borel set $K$ of finite $\mu$-measure such that

$$
\sup _{x \in E} U\left(1_{K^{c}} \mu\right)(x)<\varepsilon .
$$

We claim that there exists a $\delta>0$ such that for any Borel subset $B \subset K$ with $\mu(B)<\delta$,

$$
\left\|U\left(1_{B} \mu\right)\right\|_{\infty} \leq \varepsilon
$$

Suppose this is not true. Then one can find a sequence of Borel subsets $B_{n} \subset K$ with $\mu\left(B_{n}\right) \leq 2^{-n-1}$ so that $\left\|U\left(1_{B_{n}} \mu\right)\right\|_{\infty} \geq \varepsilon$. Define $D_{n}:=\bigcup_{k>n} B_{n}$. Then $\left\{D_{n}, n \geq 1\right\}$ is a decreasing sequence of Borel subsets with $\mu\left(D_{n}\right) \leq 2^{-n}$ so that 
$\left\|U\left(1_{D_{n}} \mu\right)\right\|_{\infty} \geq \varepsilon$. Note that $F_{n}:=\left(E \backslash D_{n}\right) \cup\left(\bigcap_{k=1}^{\infty} D_{k}\right)$ is a sequence of Borel sets that increases to $E$. Since $\mu$ is a $G$-Kato measure, $U\left(1_{D_{n}} \mu\right)=U\left(1_{E \backslash F_{n}} \mu\right)$ should decrease to zero uniformly on $E$, which is a contradiction. Therefore $\mu \in \mathbf{K}_{\infty}(X)$.

A similar proof establishes part (2) of the theorem.

It was proved in Proposition 2.1 of Chen and Song [9] that a positive smooth measure that is absolutely continuous with respect to $m$ is a $G$-Kato measure if and only if it is in $\widetilde{\mathbf{K}}_{\infty}(X)$.

Theorem 2.5. $\widetilde{\mathbf{K}}_{\infty}(X) \subset \mathbf{K}_{\infty}(X)$ and $\widetilde{\mathbf{K}}_{1}(X) \subset \mathbf{K}_{1}(X)$.

Proof. We first show that $\widetilde{\mathbf{K}}_{\infty}(X) \subset \mathbf{K}_{\infty}(X)$. It is proved in Proposition 2.1 of Chen and Song [9] that a $\widetilde{\mathbf{K}}_{\infty}(X)$-measure is a $G$-Kato measure. Thus Proposition 2.4 implies that $\widetilde{\mathbf{K}}_{\infty}(X) \subset \mathbf{K}_{\infty}(X)$.

Without loss of generality, we assume that $\mu$ is a positive measure in $\widetilde{\mathbf{K}}_{1}(X)$. From measure theory we know that $\mu$ can be decomposed as $\mu=\mu_{1}+\mu_{2}$, where $\mu_{1}$ is singular to $m$ while $\mu_{2}$ is absolutely continuous to $m$. Furthermore, there is a Borel set $F$ so that $m\left(F^{c}\right)=0$ and $\mu_{1}(F)=0$. Let $q \geq 0$ be such that $\mu_{2}(d x)=q(x) m(d x)$. By definition of $\mu \in \widetilde{\mathbf{K}}_{1}(X)$, there is a Borel set $K$ of finite $m$-mass and a constant $\delta>0$ such that

$$
\widetilde{\beta}_{1}(\mu):=\sup _{B \subset K: m(B)<\delta}\left\|U\left(1_{K^{c} \cup B} \mu\right)\right\|_{\infty}<1 .
$$

Define $F_{q}:=\{x \in F: q(x)>0\}$. Since $q$ is finite $m$-a.e., take $\eta>0$ small enough so that the set $\left\{x \in K \cap F_{q}: q(x) \leq \eta\right\}$ has $m$-mass less than $\delta / 2$. Then $K_{1}=\{x \in K \cap F: q(x)>\eta\}$ has finite $\mu$-measure while $m\left(\left(K \backslash K_{1}\right) \cap F_{q}\right) \leq \delta / 2$. Now for any Borel subset $B \subset K_{1}$ with $\mu$-mass less than $\delta_{1}=\eta \delta / 2$,

$$
m(B) \leq \eta^{-1} \int_{B} q(x) m(d x)=\eta^{-1} \mu(B)<\delta / 2 .
$$

With $B_{1}:=\left(\left(K \backslash K_{1}\right) \cap F_{q}\right) \cup F^{c}$, we have $m\left(B \cup B_{1}\right)<\delta$. Since $\mu$ does not charge on $F \backslash F_{q}$, we have

$$
\begin{aligned}
\beta_{1}(\mu) & :=\sup _{B \subset K_{1}: \mu(B)<\delta_{1}}\left\|U\left(1_{K_{1}^{c} \cup B} \mu\right)\right\|_{\infty} \\
& \leq \sup _{B \subset K_{1}: \mu(B)<\delta_{1}}\left\|U\left(1_{K^{c} \cup\left(B \cup B_{1}\right)} \mu\right)\right\|_{\infty} \\
& \leq \widetilde{\beta}_{1}(\mu)<1 .
\end{aligned}
$$

This proves that $\mu \in \mathbf{K}_{1}(X)$.

Remark 2.2. The proof for the second assertion in Theorem 2.5 can be easily modified to deduce that for measure $\mu=q m$ that is absolutely continuous with respect to $m, \mu \in \widetilde{\mathbf{K}}_{\infty}(X)$ (respectively $\mu \in \widetilde{\mathbf{K}}_{1}(X)$ ) if and only if $\mu \in \mathbf{K}_{\infty}(X)$ (respectively $\left.\mu \in \mathbf{K}_{1}(X)\right)$.

We now give two examples which show that $\mathbf{K}_{1}(X)$ is strictly larger than $\mathbf{K}_{\infty}(X)$ even when $X$ is a Brownian motion in $\mathbf{R}^{n}$.

Example 1. Let $X$ be a Brownian motion in $\mathbf{R}^{n}$ with $n \geq 3$. Let $x_{k}=\left(2^{-k}, 0\right.$, $\cdots, 0)$ in $\mathbf{R}^{n}$ and let

$$
q(x)=\sum_{k=2}^{\infty} 8^{2 k} 1_{B\left(x_{k}, 8^{-k}\right)}(x) .
$$


It was shown in Aizenman and Simon [1] as Example 1 in Appendix 1 that

$$
\lim _{r \rightarrow 0} \sup _{x \in \mathbf{R}^{d}} \int_{B(x, r)}|x-y|^{2-n} q(y) d y>0,
$$

but, nevertheless,

$$
\sup _{x \in \mathbf{R}^{d}} \int_{\mathbf{R}^{n}}|x-y|^{2-n} q(y) d y:=C_{1}<\infty .
$$

(2.12) shows that $\left(C_{1}+\varepsilon\right)^{-1} q$ is in $\mathbf{K}_{1}(X)$ for any $\varepsilon>0$ while by (2.11) $\left(C_{1}+\varepsilon\right)^{-1} q$ is not in Kato class $\mathbf{K}(X)$, not to mention in $\mathbf{K}_{\infty}(X)$.

Modifying the above example, one can easily construct a positive measure $u \in$ $\mathbf{K}_{1}(X)$ that is singular to $m=d x$ but is not in $\mathbf{K}(X)$.

Example 2. Let $X$ be a Brownian motion in $\mathbf{R}^{n}$ with $n \geq 3$. Let $x_{k}=\left(2^{-k}, 0\right.$, $\cdots, 0)$ in $\mathbf{R}^{n}$ and let measure $\mu(d x):=\sum_{k=2}^{\infty} 8^{k} \sigma_{k}(d x)$, where $\sigma_{k}(d x)$ is the surface measure for the sphere $\partial B\left(x_{k}, 8^{-k}\right)$. Then by a similar calculation as that for Example 1 in Appendix 1 of [1], we have

$$
\lim _{r \rightarrow 0} \sup _{x \in \mathbf{R}^{d}} \int_{B(x, r)}|x-y|^{2-n} \mu(d y)>0,
$$

but, nevertheless,

$$
\sup _{x \in \mathbf{R}^{d}} \int_{\mathbf{R}^{n}}|x-y|^{2-n} \mu(d y):=C_{2}<\infty .
$$

Therefore $\left(C_{2}+\varepsilon\right)^{-1} \mu$ is in $\mathbf{K}_{1}(X)$ for any $\varepsilon>0$ while $(C+\varepsilon)^{-1} \mu$ is not in Kato class $\mathbf{K}(X)$, not to mention in $\mathbf{K}_{\infty}(X)$.

2.2. Gauge Theorem for Local Perturbations. Suppose that $\mu$ is a signed smooth measure such that $\mu^{+} \in \mathbf{K}_{1}(X)$. Let $A^{+}$and $A^{-}$be the PCAFs of $X$ corresponding to $\mu^{+}$and $\mu^{-}$respectively. Clearly $A:=A^{+}-A^{-}$is the continuous additive functional of $X$ with Revuz measure $\mu$. By Proposition $2.2 A_{\zeta}^{+}$is $\mathbf{P}_{x^{-}}$ integrable. So the gauge function $g_{\mu}(x):=\mathbf{E}_{x}\left[e_{A}(\zeta)\right]$ is well defined on $E$, where $e_{A}(t)=\exp \left(A_{t}\right)$.

Theorem 2.6 (Gauge Theorem I). For smooth measure $\mu$ with $\mu^{+} \in \mathbf{K}_{1}(X)$, the gauge function $g_{\mu}$ is either bounded or identically infinite on $E$.

Proof. The proof is the same as that for Theorem 2.2 in Chen and Song [8], modulo some straightforward modifications. So it is omitted here.

Definition 2.3. Let $\mu$ be a signed smooth measure of $X$ such that $\mu^{+}$is in $\mathbf{K}_{1}(X)$. We say $(X, \mu)$ is gaugeable if the gauge function $x \mapsto \mathbf{E}_{x}\left[e_{A}(\zeta)\right]$ is bounded on $E$.

Remark 2.3. Note that the definitions of $\mathbf{K}_{\infty}(X)$ and $\mathbf{K}_{1}(X)$ are time-change invariant, in contrast to the Kato class $\mathbf{K}(X)$. That is, if $C$ is a strictly increasing PCAF of $X$ and if $X_{C^{-1}}$ is the associated time-changed process (more precisely, $C^{-1}=\left\{\tau_{t}, t \geq 0\right\}$ with $\left.\tau_{t}=\inf \left\{s: C_{t}>t\right\}\right)$, then $\mathbf{K}_{\infty}\left(X_{C^{-1}}\right)=\mathbf{K}_{\infty}(X)$ and $\mathbf{K}_{1}\left(X_{C^{-1}}\right)=\mathbf{K}_{1}(X)$. This is because if one uses the Revuz measure of $C$ as the reference measure for $X_{C^{-1}}$, then by Fitzsimmons and Getoor 19], processes $X$ and $X_{C^{-1}}$ have the same class of smooth measures. Furthermore, if $\mu$ is a smooth measure of $X$ with associated PCAF $A$ of $X$, then $A_{C^{-1}}$ is the PCAF of 
$X_{C^{-1}}$ with the same Revuz measure $\mu$. Therefore the potential $U \mu$ of $\mu$ is timechange invariant; so are $\mathbf{K}_{\infty}(X)$ and $\mathbf{K}_{1}(X)$. In view of Proposition [2.3(1), we have $\mathbf{K}_{\infty}(X) \subset \bigcap_{C} \mathbf{K}\left(X_{C^{-1}}\right)$.

On the other hand, the conclusion of the gauge theorem as well as its hypotheses are also time-change invariant.

We now give some equivalent conditions for $(X, \mu)$ to be gaugeable. The following two results should be compared with Lemma 9 and Theorem 4 in Chung and Rao [12 respectively. Here a suitable sequence of stopping times is used for time partition as opposed to a sequence of (deterministic) constants used in [12]. Suppose that $\mu$ is a signed smooth measure with $\mu^{+} \in \mathbf{K}_{1}(X)$ and $U \mu^{-}$bounded. Let $A^{+}$and $A^{-}$be the PCAFs of $X$ with Revuz measures $\mu^{+}$and $\mu^{-}$respectively. Let $\tau_{t}$ be the right continuous inverse of $A_{t}^{+}$; that is,

$$
\tau_{t}=\inf \left\{s: A_{s}^{+}>t\right\}
$$

with the convention that $\inf \emptyset=\infty$.

Lemma 2.7. Suppose that $\mu$ is a signed smooth measure such that $\mu^{+} \in \mathbf{K}_{1}(X)$ and $U \mu^{-}$is bounded. If $(X, \mu)$ is gaugeable, then for any $\delta>0$ there is a constant $c(\delta)>0$ such that

$$
\left\|g_{\mu}\right\|_{\infty}^{-1} g_{\mu}(x) \leq \sum_{n=0}^{\infty} \mathbf{E}_{x}\left[e_{A}\left(\tau_{n \delta}\right) ; \tau_{n \delta}<\zeta\right] \leq c(\delta)<\infty \quad \text { for all } x \in E .
$$

Proof. Since the proof is the same for any $\delta>0$, we take $\delta=1$. Let $S:=\{x \in$ $\left.E: \mathbf{P}_{x}\left(\tau_{0}=0\right)=1\right\}$ be the fine support of $\mu^{+}$. The time-changed process $Y_{t}$ of $X_{t}$ by $A^{+}$is defined by $Y_{t}=X_{\tau_{t}}$. This time-changed process $Y$ is a right process on state space $S$ in the sense of Sharpe [33] with lifetime $\zeta^{Y}=A_{\zeta}^{+}$. Note that for Borel $f \geq 0$,

$$
\begin{aligned}
\mathbf{E}_{x}\left[\int_{0}^{\infty} f\left(Y_{t}\right) d t\right] & =\mathbf{E}_{x}\left[\int_{0}^{\infty} f\left(X_{\tau_{t}}\right) d t\right] \\
& =\mathbf{E}_{x}\left[\int_{0}^{\infty} f\left(X_{t}\right) d A_{t}^{+}\right]=U\left(f \mu^{+}\right)(x) .
\end{aligned}
$$

Since $\mu^{+} \in \mathbf{K}_{1}(X)$, there is $p>1$ such that $p \mu^{+} \in \mathbf{K}_{1}(X)$. According to the definition for $p \mu^{+} \in \mathbf{K}_{1}(X)$, there is a Borel set $K_{1}$ of finite $\mu^{+}$-mass and a constant $\delta>0$ such that

$$
\sup _{B \subset K_{1}: \mu^{+}(B)<\delta}\left\|U\left(1_{K_{1}^{c} \cup B} \mu^{+}\right)\right\|_{\infty}:=\beta_{1}<1 .
$$

Since $(X, \mu)$ is gaugeable, $g_{\mu}(x):=\mathbf{E}_{x}\left[e_{A}(\zeta)\right]$ is bounded and so

$$
\lim _{n \rightarrow \infty} \mathbf{E}_{x}\left[e_{A}(\zeta) ; \tau_{n}<\zeta\right]=0 \text { on } E .
$$

Thus for any given small $\varepsilon>0$ one can find an integer $N$ large enough and a closed subset $K \subset K_{1}$ such that

$$
\sup _{x \in K} \mathbf{E}_{x}\left[e_{A}(\zeta) ; \tau_{N}<\zeta\right]<\varepsilon,
$$

and $\mu^{+}\left(K_{1} \backslash K\right)<\delta$. Note that by (2.16),

$$
p\left\|U\left(1_{K^{c}} \mu^{+}\right)\right\|_{\infty} \leq \beta_{1}<1 .
$$


Using Khasminskii's lemma,

$$
\sup _{x \in E} \mathbf{E}_{x}\left[e_{A}\left(\sigma_{K}\right)^{p}\right] \leq \sup _{x \in E} \mathbf{E}_{x}\left[\exp \left(p A_{\sigma_{K}}^{+}\right)\right] \leq \frac{1}{1-p\left\|U\left(1_{K^{c}} \mu^{+}\right)\right\|_{\infty}} \leq\left(1-\beta_{1}\right)^{-1},
$$

where $\sigma_{K}$ is the first hitting time of $K$ by $X$. Now

$$
\begin{aligned}
\mathbf{E}_{x}\left[e_{A}(\zeta) ; \tau_{2 N}<\zeta\right] \\
\leq \mathbf{E}_{x}\left[e_{A}(\zeta) ; \tau_{N}<\sigma_{K}\right]+\mathbf{E}_{x}\left[e_{A}(\zeta) ; \sigma_{K} \leq \tau_{N}<\tau_{2 N}<\zeta\right] \\
\leq\left\|g_{\mu}\right\|_{\infty} \mathbf{E}_{x}\left[e_{A}\left(\sigma_{K}\right) ; \tau_{N}<\sigma_{K}\right] \\
\quad+\mathbf{E}_{x}\left[e_{A}\left(\sigma_{K}\right) \mathbf{E}_{X_{\sigma_{K}}}\left[e_{A}(\zeta) ; \tau_{N}<\zeta\right] ; \sigma_{K}<\zeta\right] \\
\quad:=I+I I
\end{aligned}
$$

Denote by $\mathbf{P}_{x}^{Y}$ the probability law for the time-changed process $Y$ and let $\sigma_{K}^{Y}$ be the first hitting time of the set $K$ (more precisely $K \cap S$ ) by $Y$. It follows from (2.15) that

$$
\mathbf{E}_{x}^{Y}\left[\sigma_{K}^{Y} \wedge \zeta^{Y}\right] \leq \mathbf{E}_{x}^{Y}\left[\int_{0}^{\infty} 1_{K^{c}}\left(Y_{t}\right) d t\right]=U\left(1_{K^{c}} \mu^{+}\right)(x)
$$

Let $q>1$ be such that $\frac{1}{p}+\frac{1}{q}=1$ and use Hölder's inequality,

$$
\begin{aligned}
I & \leq\left\|g_{\mu}\right\|_{\infty}\left(\mathbf{E}_{x}\left[e_{A}\left(\sigma_{K}\right)^{p}\right]\right)^{1 / p} \mathbf{P}_{x}\left(\tau_{N}<\sigma_{K}\right)^{1 / q} \\
& \leq\left(1-\beta_{1}\right)^{-1 / p}\left\|g_{\mu}\right\|_{\infty} \mathbf{P}_{x}^{Y}\left(N<\sigma_{K}^{Y}\right)^{1 / q} \\
& \leq \frac{\left\|g_{\mu}\right\|_{\infty}\left(\mathbf{E}_{x}^{Y}\left[\sigma_{K}^{Y} \wedge \zeta^{Y}\right]\right)^{1 / q}}{\left(1-\beta_{1}\right)^{1 / p} N^{1 / q}} \leq \frac{\left\|g_{\mu}\right\|_{\infty}\left(U\left(1_{K^{c}} \mu^{+}\right)(x)\right)^{1 / q}}{\left(1-\beta_{1}\right)^{1 / p} N^{1 / q}} \\
& \leq \frac{\left(\beta_{1} / p\right)^{1 / q}\left\|g_{\mu}\right\|_{\infty}}{\left(1-\beta_{1}\right)^{1 / p} N^{1 / q}} .
\end{aligned}
$$

So $I \rightarrow 0$ uniformly as $N \rightarrow \infty$. On the set $\left\{\sigma_{K}<\zeta\right\}, X_{\sigma_{K}} \in K$ and so

$$
I I \leq \varepsilon \mathbf{E}_{x}\left[e_{A^{+}}\left(\sigma_{K}\right) ; \sigma_{K}<\zeta\right] \leq \frac{\varepsilon}{1-\left\|U\left(1_{K^{c}} \mu^{+}\right)\right\|_{\infty}} \leq \frac{\varepsilon}{1-\beta_{1}}
$$

Thus for large $N, \sup _{x \in E} \mathbf{E}_{x}\left[e_{A}(\zeta) ; \tau_{N}<\zeta\right]$ is small. By Jensen's inequality and the fact that by Proposition $2.2 U|\mu|$ is bounded, the gauge function $g_{\mu}$ is bounded from below by a positive constant, say $c_{1}$. So

$\mathbf{E}_{x}\left[e_{A}\left(\tau_{N}\right) ; \tau_{N}<\zeta\right] \leq c_{1}^{-1} \mathbf{E}_{x}\left[e_{A}\left(\tau_{N}\right) g_{\mu}\left(X_{\tau_{N}}\right) ; \tau_{N}<\zeta\right] \leq c_{1}^{-1} \mathbf{E}_{x}\left[e_{A}(\zeta) ; \tau_{N}<\zeta\right]$

Therefore, when $N$ is large enough,

$$
\sup _{x \in E} \mathbf{E}_{x}\left[e_{A}\left(\tau_{N}\right) ; \tau_{N}<\zeta\right]:=\lambda<1 .
$$

By the strong Markov property of $X$,

$$
\sup _{x \in E} \mathbf{E}_{x}\left[e_{A}\left(\tau_{k N}\right) ; \tau_{k N}<\zeta\right] \leq \lambda^{k}, \quad \text { for } k \geq 0 .
$$

Note that

$$
\mathbf{E}_{x}\left[e_{A}\left(\tau_{j}\right) ; \tau_{j}<\zeta\right] \leq \mathbf{E}_{x}\left[e_{A^{+}}\left(\tau_{j}\right) ; \tau_{j}<\zeta\right] \leq e^{j}
$$


Let $M:=\sum_{j=0}^{N-1} e^{j}$. Then for $0 \leq j<N$,

$$
\begin{aligned}
\mathbf{E}_{x}\left[e_{A}\left(\tau_{j+k N}\right) ; \tau_{j+k N}<\zeta\right] & =\mathbf{E}_{x}\left[e_{A}\left(\tau_{j}\right) \mathbf{E}_{X_{\tau_{j}}}\left(e_{A}\left(\tau_{k N}\right) ; \tau_{k N}<\zeta\right) ; \tau_{j}<\zeta\right] \\
& \leq \lambda^{k} \max _{0 \leq j<N} \mathbf{E}_{x}\left[e_{A}\left(\tau_{j}\right) ; \tau_{j}<\zeta\right] \leq \lambda^{k} M .
\end{aligned}
$$

All these inequalities lead to

$$
\begin{aligned}
\sum_{n=0}^{\infty} \mathbf{E}_{x}\left[e_{A}\left(\tau_{n}\right) ; \tau_{n}<\zeta\right] & =\sum_{k=0}^{\infty} \sum_{j=0}^{N-1} \mathbf{E}_{x}\left[e_{A}\left(\tau_{j+k N}\right) ; \tau_{k N+j}<\zeta\right] \\
& \leq N M \sum_{k=0}^{\infty} \lambda^{k}=N M(1-\lambda)^{-1}<\infty
\end{aligned}
$$

Finally, we have, by the strong Markov property of $X$,

$$
\begin{aligned}
g_{\mu}(x) & =\sum_{n=0}^{\infty} \mathbf{E}_{x}\left[e_{A}(\zeta) ; \tau_{n}<\zeta \leq \tau_{n+1}\right] \\
& \leq \sum_{n=0}^{\infty} \mathbf{E}_{x}\left[e_{A}\left(\tau_{n}\right) g_{\mu}\left(X_{\tau_{n}}\right) ; \tau_{n}<\zeta\right] \\
& \leq\left\|g_{\mu}\right\|_{\infty} \sum_{k=0}^{\infty} \mathbf{E}_{x}\left[e_{A}\left(\tau_{n}\right) ; \tau_{n}<\zeta\right] .
\end{aligned}
$$

This proves the lemma.

Remark 2.4. If $E$ is Green-bounded, that is, if $\|G 1\|_{\infty}<\infty$, then an easy modification (without doing time change) of the above proof shows the following (cf. Lemma 9 of Chung and Rao [12]).

Suppose that $\mu$ is a signed smooth measure such that $\mu^{+} \in \mathbf{K}_{1}(X)$ and $U \mu^{-}$is bounded. If $(X, \mu)$ is gaugeable and $\|G 1\|_{\infty}<\infty$, then for any $\delta>0$ there is a constant $c(\delta)>0$ such that

$$
\left\|g_{\mu}\right\|_{\infty}^{-1} g_{\mu}(x) \leq \sum_{n=0}^{\infty} \mathbf{E}_{x}\left[e_{A}(n \delta) ; n \delta<\zeta\right] \leq c(\delta)<\infty \quad \text { for all } x \in E .
$$

Theorem 2.8. Suppose that $\mu$ is a signed smooth measure such that $\mu^{+} \in \mathbf{K}_{1}(X)$ and $U \mu^{-}$is bounded. Let $A^{+}$and $A^{-}$be the PCAFs with Revuz measures $\mu^{+}$and $\mu^{-}$respectively. If $(X, \mu)$ is gaugeable, then

$$
\sup _{x \in E} \mathbf{E}_{x}\left[\int_{0}^{\zeta} e_{A}(t) d A_{t}^{+}\right]<\infty .
$$

Proof. Note that

$$
\int_{0}^{t} e_{A}(s) d A_{s}^{+} \leq \int_{0}^{t} e_{A^{+}}(s) d A_{s}^{+}=\exp \left(A_{t}^{+}\right)-1
$$

and by the definition of $\tau_{t}$,

$$
C(t):=\sup _{x \in E} \mathbf{E}_{x}\left[\exp \left(A_{\tau_{t}}^{+}\right)\right] \leq e^{t}<\infty .
$$


Now

$$
\begin{aligned}
\mathbf{E}_{x}\left[\int_{0}^{\zeta} e_{A}(t) d A_{t}^{+}\right] & =\mathbf{E}_{x}\left[\sum_{n=0}^{\infty} \int_{\tau_{n} \wedge \zeta}^{\tau_{n+1} \wedge \zeta} e_{A}(t) d A_{t}^{+}\right] \\
& =\sum_{n=0}^{\infty} \mathbf{E}_{x}\left[\int_{\tau_{n}}^{\tau_{n+1} \wedge \zeta} e_{A}(t) d A_{t}^{+} ; \tau_{n}<\zeta\right] \\
& =\sum_{n=0}^{\infty} \mathbf{E}_{x}\left[e_{A}\left(\tau_{n}\right) \mathbf{E}_{X_{\tau_{n}}}\left(\int_{0}^{\tau_{1} \wedge \zeta} e_{A}(t) d A_{t}^{+}\right) ; \tau_{n}<\zeta\right] \\
& \leq C(1) \sum_{n=0}^{\infty} \mathbf{E}_{x}\left[e_{A}\left(\tau_{n}\right): \tau_{n}<\zeta\right],
\end{aligned}
$$

which is bounded on $E$ by Lemma 2.7

Remark 2.5. The above arguments can be easily modified, with $\tau_{t}$ being defined to be the right continuous inverse of $|A|_{t}$ as opposed to $A_{t}^{+}$, to show that if $\mu$ is a signed measure in $\mathbf{K}_{1}(X)$ such that $(X, \mu)$ is gaugeable, then

$$
\sup _{x \in E} \mathbf{E}_{x}\left[\int_{0}^{\zeta} e_{A}(t) d|A|_{t}\right]<\infty,
$$

where $|A|=A^{+}+A^{-}$is the PCAF corresponding to the Revuz measure $|\mu|=$ $\mu^{+}+\mu^{-}$.

Corollary 2.9. Suppose that $\mu$ is a signed smooth measure such that $\mu^{+} \in \mathbf{K}_{1}(X)$ and $U \mu^{-}$is bounded. Let $A^{+}$and $A^{-}$be the PCAFs of $X$ with Revuz measures $\mu^{+}$ and $\mu^{-}$respectively. Then the following are equivalent:

(1) $(X, \mu)$ is gaugeable;

(2) $\mathbf{E}_{x}\left[\int_{0}^{\zeta} e_{A}(t) d A_{t}^{+}\right]<\infty$ for some $x \in E$;

(3) $\sup _{x \in E} \mathbf{E}_{x}\left[\int_{0}^{\zeta} e_{A}(t) d A_{t}^{+}\right]<\infty$;

(4) $\mathbf{E}_{x}\left[\sup _{t \leq \zeta} e_{A}(t)\right]<\infty$ for some $x \in E$;

(5) $\sup _{x \in E} \mathbf{E}_{x}\left[\sup _{t \leq \zeta} e_{A}(t)\right]<\infty$.

Proof. Since

$$
\int_{0}^{\zeta} e_{A}(t) d A_{t}^{+}=\int_{0}^{\zeta} e^{-A_{t}^{-}} d e^{A_{t}^{+}} \geq e^{-A_{\zeta}^{-}}\left(e^{A_{\zeta}^{+}}-1\right) \geq e_{A}(\zeta)-1,
$$

the equivalence between (1)-(3) follows immediately from Theorems 2.6 and 2.8 For the remaining, observe that

$$
e_{A}(t)=1+\int_{0}^{t} e_{A}(s) d A_{s} \leq 1+\int_{0}^{t} e_{A}(s) d A_{s}^{+},
$$

and so

$$
e_{A}(\zeta) \leq \sup _{0 \leq t \leq \zeta} e_{A}(t) \leq 1+\int_{0}^{\zeta} e_{A}(s) d A_{s}^{+} .
$$

The equivalence between (1)-(5) now follows. 
We now present a result that will be useful in deriving an analytic characterization of $(X, \mu)$ being gaugeable in section 5 .

Suppose $\mu$ is a signed smooth measure such that $\mu^{+} \in \mathbf{K}_{1}(X)$ and $U \mu^{-}$is bounded. Let $A^{+}$and $A^{-}$be the PCAFs of $X$ with Revuz measures $\mu^{+}$and $\mu^{-}$ respectively. Let $Y$ be the subprocess of $X$ killed at rate $\mu^{-}$; in other words, $Y$ is obtained from $X$ through the Feynman-Kac transform $e^{-A_{t}^{-}}$. It is known (cf. Sharpe [33]) that $Y$ is a right process, which is clearly transient and irreducible. By Fitzsimmons and Getoor [19], $X$ and $Y$ have the same class of smooth measures, in particular, $A^{+}$can be viewed as the continuous additive functional of $Y$ with Revuz measure $\mu^{+}$. It is clear that the potential operator $U^{Y}$ of $Y$ satisfies $U^{Y} \leq U$. Therefore $\mu^{+} \in \mathbf{K}_{1}(X) \subset \mathbf{K}_{1}(Y)$ and $U^{Y} \mu^{-}$is bounded.

Theorem 2.10. Under the notation of the last paragraph, $(X, \mu)$ is gaugeable if and only if $\left(Y, \mu^{+}\right)$is gaugeable.

Proof. We have by $\mathbf{6 2}$ of Sharpe [33] and integration by parts,

$$
\begin{aligned}
\mathbf{E}_{x}^{Y}\left[e^{A_{\zeta}^{+}}\right] & =\mathbf{E}_{x}\left[\int_{0}^{\zeta} e^{A_{s}^{+}} d\left(-e^{-A_{s}^{-}}\right)+e^{A_{\zeta}^{+}} e^{-A_{\zeta}^{-}}\right] \\
& =\mathbf{E}_{x}\left[\int_{0}^{\zeta} e^{-A_{s}^{-}} d e^{A_{s}^{+}}\right]+1 \\
& =1+\mathbf{E}_{x}\left[\int_{0}^{\zeta} e_{A}(t) d A_{t}^{+}\right] .
\end{aligned}
$$

Thus by Corollary 2.9, $(X, \mu)$ is gaugeable if and only if $\left(Y, \mu^{+}\right)$is gaugeable.

The following result can be obtained from Remark 2.4 using the same argument as that for Theorem 6 in Chung and Rao 12. It will be applied to give a characterization of the gaugeability of $(X, \mu)$ in terms the positivity of the $L^{2}$-spectral radius of the corresponding Schrödinger semigroup $Q_{t}$ :

$$
Q_{t} f(x):=\mathbf{E}_{x}\left[e_{A}(t) f\left(X_{t}\right)\right] .
$$

Theorem 2.11. Suppose that $\|G 1\|_{\infty}<\infty$ and that $\mu$ is a signed smooth measure such that $\mu^{+} \in \mathbf{K}_{1}(X)$ and $U \mu^{-}$is bounded. Then the following are equivalent:

(1) $(X, \mu)$ is gaugeable.

(2) For some $\delta>0$ and some $x \in E$,

$$
\sum_{n=1}^{\infty} Q_{n \delta} 1(x)<\infty
$$

(3) For all $\delta>0$ and all $x \in E$, (2.20) is true.

(4) For some $x \in E, \int_{0}^{\infty} Q_{t} 1(x) d t<\infty$.

(5) There exists some $t>0$ such that $\left\|Q_{t} 1\right\|_{\infty}<1$.

(6) There are constants $C>0$ and $b>0$ such that $\left\|Q_{t} 1\right\|_{\infty} \leq C e^{-b t}$ for $t>0$.

For the next result in this section, we assume in addition that $X$ is $m$-symmetric. Note that in this case, the Revuz formula (2.2) holds. Let $\mu$ be a signed smooth measure such that $\mu^{+} \in \mathbf{K}_{1}(X)$ and $U \mu^{-}$is bounded. Note that semigroup $Q_{t}$ can be viewed as the Feynman-Kac semigroup of $Y$ by the transformation $e^{A_{t}^{+}}$, where $Y$ is the subprocess of $X$ killed at rate $\mu^{-}$as mentioned above. So it follows 
from Proposition 2.3] and Stollmann and Voigt [36] (see also Getoor [24]) that the Schrödinger semigroup $Q_{t}$ is strongly continuous in $L^{p}(E, m)$ for every $1 \leq p<\infty$. If we use $\mathcal{L}$ to denote the infinitesimal generator for the semigroup of $X$, then the infinitesimal generator for the semigroup $Q_{t}$ is $\mathcal{L}+\mu$. For $1 \leq p \leq \infty$, define the $L^{p}$-spectral radius of the semigroup $Q_{t}$ by

$$
\lambda_{p}(X, \mu):=-\lim _{t \rightarrow \infty} \frac{1}{t} \log \left\|Q_{t}\right\|_{p, p}
$$

Here $\left\|Q_{t}\right\|_{p, p}$ is the operator norm of $Q_{t}: L^{p}(E, m) \rightarrow L^{p}(E, m)$. The $L^{p}$-norm in $L^{p}(E, m)$ will be denoted as $\|\cdot\|_{p}$. Clearly

$$
\left\|Q_{t}\right\|_{\infty, \infty}=\left\|Q_{t} 1\right\|_{\infty}=\sup _{x \in E} \mathbf{E}_{x}\left[e_{A}(t)\right]
$$

Since $X$ is symmetric, the semigroup $Q_{t}$ is symmetric in $L^{2}(E, m)$. Let $(\mathcal{E}, \mathcal{F})$ be the Dirichlet space of $X$. Then the quadratic form associated with $Q_{t}$ is given by $(\mathcal{Q}, \mathcal{F})$ (see Chen and Song $[9]$ ), where

$$
\mathcal{Q}(u, v)=\mathcal{E}(u, v)-\int_{E} u(x) v(x) \mu(d x) .
$$

It is well known that the $L^{2}$-spectral radius $\lambda_{2}(X, \mu)$ of $Q_{t}$ can be represented in terms of its quadratic form $\mathcal{Q}$ :

$$
\begin{aligned}
\lambda_{2}(X, \mu) & =\inf \left\{\mathcal{Q}(u, u): u \in \mathcal{F} \text { with } \int_{E} u(x)^{2} m(d x)=1\right\} \\
& =\inf \left\{\mathcal{E}(u, u)-\int_{E} u(x)^{2} \mu(d x): u \in \mathcal{F} \text { with } \int_{E} u(x)^{2} m(d x)=1\right\} .
\end{aligned}
$$

Theorem 2.12. In addition to the assumption made at the beginning of this section, $X$ is further assumed to be m-symmetric. Suppose that $m(E)<\infty$ and $\|G 1\|_{\infty}<\infty$. Let $\mu$ be a signed smooth measure such that $\mu^{+} \in \mathbf{K}_{1}(X)$ and $U \mu^{-}$ is bounded. Then $(X, \mu)$ is gaugeable if and only if $\lambda_{2}(X, \mu)>0$. Furthermore, if $\lambda_{2}(X, \mu)>0$, then $\lambda_{p}(X, \mu)$ is independent of $p \in[1, \infty]$.

Proof. It follows from the duality and interpolation that

$$
\left\|Q_{t}\right\|_{1,1}=\left\|Q_{t}\right\|_{\infty, \infty} \quad \text { and } \quad\left\|Q_{t}\right\|_{2,2} \leq\left\|Q_{t}\right\|_{p, p} \leq\left\|Q_{t}\right\|_{\infty, \infty} \quad \text { for } 1<p<\infty .
$$

Hence

$$
\lambda_{\infty}(X, \mu) \leq \lambda_{p}(X, \mu) \leq \lambda_{2}(X, \mu) \text { for } 1<p<\infty
$$

By Theorem 2.11, if $(X, \mu)$ is gaugeable, then $\lambda_{\infty}(X, \mu)>0$ and therefore $\lambda_{2}(X, \mu)$ $>0$. Conversely, suppose $\lambda_{2}(X, \mu)>0$. Then for any $\varepsilon \in\left(0, \lambda_{2}(X, \mu)\right)$, there is $t_{0}(\varepsilon)>0$ such that

$$
\left\|Q_{t}\right\|_{2,2} \leq e^{-t\left(\lambda_{2}(X, \mu)-\varepsilon\right)} \quad \text { for } t \geq t_{0}(\varepsilon) .
$$

Since $1 \in L^{2}(E, m), \int_{0}^{\infty} Q_{t} 1 d t$ is $L^{2}(E, m)$-integrable. Hence by Theorem 2.11 $(X, \mu)$ is gaugeable. Now suppose $\lambda_{2}(X, \mu)>0$. Then for any $\varepsilon \in\left(0, \lambda_{2}(X, \mu)\right)$, by $(2.22)$,

$$
\left\|Q_{t}\right\|_{\infty, \infty}=\left\|Q_{t} 1\right\|_{\infty} \leq m(E) e^{-t\left(\lambda_{2}(X, \mu)-\varepsilon\right)} \quad \text { for } t \geq t_{0}(\varepsilon) .
$$

This implies that $\lambda_{\infty}(X, \mu) \geq \lambda_{2}(X, \mu)-\varepsilon$ and so $\lambda_{\infty}(X, \mu) \geq \lambda_{2}(X, \mu)$. Hence by (2.21),$\lambda_{\infty}(X, \mu)=\lambda_{2}(X, \mu)=\lambda_{p}(X, \mu)$ for all $p \in[1, \infty]$. 
See Chung and Zhao [13] and Chen and Song [7] for the characterization of gaugeability of $(X, \mu)$ in terms of the condition $\lambda_{2}(X, \mu)>0$ when $X$ is a Brownian motion and a symmetric stable process in a Euclidean domain, respectively, and Simon [35] and the references therein for the $L^{p}$-independence of spectral radius $\lambda_{p}(X, \mu)$ where $X$ is a Brownian motion in $\mathbf{R}^{n}$.

2.3. Gauge Theorem for Non-local Perturbations. In Chen and Song 9, gauge and conditional gauge theorems were established for discontinuous additive functionals of $X$. We now introduce a refined Kato class $\mathbf{J}_{\infty}(X)$ for discontinuous additive functionals and present its gauge theorem.

In this subsection, $X$ is further assumed to be a Borel standard process on $E$, instead of being a Borel right process. Here, a Borel standard process on the Lusin space $E$ is a strong Markov process satisfying the following conditions: (i) it is right continuous, (ii) it has no branching points, (iii) its resolvents map Borel functions into Borel functions and (iv) it is quasi-left continuous on $(0, \zeta)$, where $\zeta$ is the lifetime of the process. This additional assumption is to ensure the existence of a Lévy system $(N, H)$ for $X$ (see Benveniste and Jacod [3] and Theorem 47.10 of Sharpe [33]). Here $H$ is a PCAF of $X$ with bounded 1-potential, and $N(x, d y)$ is a kernel on $\left(E_{\partial}, \mathcal{B}\left(E_{\partial}\right)\right)$ with $N(x,\{x\})=0$ for all $x \in E$ such that for any nonnegative Borel function $F$ on $E \times E_{\partial}$ vanishing on the diagonal and any $x \in E$, $t \mapsto \int_{0}^{t}\left(\int_{E_{\partial}} F\left(X_{s}, y\right) N\left(X_{s}, d y\right)\right) d H_{s}$ is the dual predictable projection of $t \mapsto$ $\sum_{s \leq t} F\left(X_{s-}, X_{s}\right)$ under every $\mathbf{P}_{x}$; that is,

$$
\mathbf{E}_{x}\left[\sum_{s \leq t} F\left(X_{s-}, X_{s}\right)\right]=\mathbf{E}_{x}\left[\int_{0}^{t} \int_{E_{\partial}} F\left(X_{s}, y\right) N\left(X_{s}, d y\right) d H_{s}\right] .
$$

The Revuz measure of $H$ will be denoted as $\mu_{H}$. To simplify notation, we write

$$
N F(x):=\int_{E_{\partial}} F(x, y) N(x, d y) .
$$

Definition 2.4. Suppose $F$ is a bounded function on $E \times E$ vanishing on the diagonal. We say that $F$ belongs to the class $\mathbf{J}_{\infty}(X)$ if the measure

$$
\mu_{|F|}(d x):=N|F|(x) \mu_{H}(d x)
$$

belongs to $\mathbf{K}_{\infty}(X)$.

In Chen and Song [9], the corresponding Kato class, which we now denote as $\widetilde{\mathbf{J}}_{\infty}(X)$, is the family of those bounded functions $F$ with $\mu_{|F|} \in \widetilde{\mathbf{K}}_{\infty}(X)$. As already seen, $\mathbf{J}_{\infty}(X)$ is an extension of $\widetilde{\mathbf{J}}_{\infty}(X)$ in that the Revuz measure $\mu_{H}$ does not need to be absolutely continuous with respect to $m$.

For a smooth measure $\mu$ associated with a continuous additive functional $A^{\mu}$ and a Borel function $F$ on $E \times E$ that vanishes along the diagonal, define

$$
A_{t}^{F}:=\sum_{0<s \leq t} F\left(X_{s-}, X_{s}\right) \quad \text { and } \quad e_{A^{\mu}+F}(t):=\exp \left(A_{t}^{\mu}+A_{t}^{F}\right), \quad t \geq 0 .
$$

When $\mu \in \mathbf{K}_{1}(X)$ and $F \in \mathbf{J}_{\infty}(X)$, by Proposition 2.2 and the definition of $\mathbf{J}_{\infty}(X)$,

$$
\sup _{x \in E} \mathbf{E}_{x}\left[A_{\zeta}^{|\mu|}+A_{\zeta}^{|F|}\right]<\infty
$$


and so the gauge function $g(x):=\mathbf{E}_{x}\left[e_{A^{\mu}+F}(\zeta)\right]$ is well defined. Furthermore, by Jensen's inequality,

$$
\inf _{x \in E} \mathbf{E}_{x}\left[e_{A^{\mu}+F}(\zeta)\right]>0 .
$$

Theorem 2.13 (Gauge Theorem II). Assume that a signed measure $\mu \in \mathbf{K}_{1}(X)$ and $F \in \mathbf{J}_{\infty}(X)$. Then the gauge function $g(x):=\mathbf{E}_{x}\left[e_{A^{\mu}+F}(\zeta)\right]$ is either bounded on $E$ or identically $\infty$ on $E$.

Proof. The proof is the same as that for Theorem 2.6 in Chen and Song [9], modulo some suitable modifications. So it is omitted here.

Definition 2.5. For $\mu \in \mathbf{K}_{1}(X)$ and $F \in \mathbf{J}_{\infty}(X)$, we say $\left(X, A^{\mu}+F\right)$ is gaugeable if the gauge function $x \mapsto \mathbf{E}_{x}\left[e_{A^{\mu}+F}(\zeta)\right]$ is bounded on $E$. Here $A^{\mu}$ is the continuous additive functional of $X$ with Revuz measure $\mu$.

The Feynman-Kac transform (2.23) defines a semigroup

$$
Q_{t} f(x):=\mathbf{E}_{x}\left[e_{A^{\mu}+F}(t) f\left(X_{t}\right)\right] .
$$

As suggested in Theorem 2.4 of Chen and Song [9], $\left\{Q_{t}, t \geq 0\right\}$ is a semigroup having infinitesimal generator of the form $\mathcal{L}+\mu_{H} \mathbf{F}+\mu$, where $\mathcal{L}$ is the infinitesimal generator of the semigroup of $X$ and $\mathbf{F}$ is a non-local linear operator defined by

$$
\mathbf{F} f(x):=\int_{E_{\partial}}\left(e^{F(x, y)}-1\right) f(y) N(x, d y), \quad x \in E .
$$

So the Schrödinger semigroup in (2.25) defines a non-local perturbation of $X$ by discontinuous additive functionals $A^{\mu}+A^{F}$.

The results established above for $(X, \mu)$ from Lemma 2.7 to Theorem 2.12 can all be extended to the non-local perturbation $\left(X, A^{\mu}+F\right)$, along the same line of arguments with suitable modifications. We now present these generalizations. First let us recall the definition of the Stieltjes exponential $\operatorname{Exp}(K)_{t}$ of a right continuous increasing function $K_{t}$ on $\mathbf{R}_{+}$with $K_{0}=1 \cdot \operatorname{Exp}(K)_{t}$ is the unique solution $Z_{t}$ of

$$
Z_{t}=1+\int_{(0, t]} Z_{s-} d K_{s}, \quad t>0 .
$$

By (A4.17) of Sharpe 33,

$$
\operatorname{Exp}\left(K_{t}\right)=e^{K_{t}^{c}} \prod_{0<s \leq t}\left(1+\Delta K_{s}\right)
$$

where $\Delta K_{s}=K_{s}-K_{s-}$ and $K_{t}^{c}$ denotes the continuous part of $K_{t}$. Clearly $\exp \left(K_{t}\right) \geq \operatorname{Exp}(K)_{t}$ with the equality holding if and only if $K_{t}$ is continuous. The reason for $\operatorname{Exp}(K)_{t}$ being called the Stieltjes exponential of $K_{t}$ is that by DoléansDade [16] it can be expressed as

$$
\operatorname{Exp}(K)_{t}=1+\sum_{n=1}^{\infty} \int_{(0, t]} d K_{t_{n}} \int_{\left(0, t_{n}\right]} d K_{t_{n-1}} \cdots \int_{\left(0, t_{2}\right]} d K_{t_{1}} .
$$

The advantage of using the Stieltjes exponential $\operatorname{Exp}(K)_{t}$ over the regular exponential $\exp \left(K_{t}\right)$ is that the Khasminskii inequality continues to hold for Stieltjes exponentials in view of (2.27). It is stated as follows. If $\sup _{x \in E} \mathbf{E}_{x}\left[K_{t}\right]<1$, then (see Lemma 2.1 of Ying [41])

$$
\mathbf{E}_{x}\left[\operatorname{Exp}(K)_{t}\right] \leq \frac{1}{1-\sup _{x \in E} \mathbf{E}_{x}\left[K_{t}\right]}
$$


In Stummer and Sturm 37, a refined version of Khasminskii's inequality is given.

Suppose that $\mu \in \mathbf{K}_{1}(X)$ and $F \in \mathbf{J}_{\infty}(X)$. Let $A^{+}$and $A^{-}$denote the PCAFs of $X$ with Revuz measures $\mu^{+}$and $\mu^{-}$respectively, and let $F^{+}$and $F^{-}$denote the positive and negative parts of $F$ respectively. Recall that $\mu_{F^{+}}(d x):=N F^{+}(x) \mu_{H}(d x)$. Let $\tau_{t}$ be the right continuous inverse of the PCAF corresponding to the smooth measure $\mu^{+}+\mu_{F^{+}}$, that is,

$$
\tau_{t}=\inf \left\{s \geq 0: A_{s}^{+}+\int_{0}^{s} N F^{+}\left(X_{r}\right) d H_{r}>t\right\} \quad \text { for } t \geq 0 .
$$

Lemma 2.14. Suppose that $\mu$ is a signed measure in $\mathbf{K}_{1}(X)$ and $F \in \mathbf{J}_{\infty}(X)$. Let $A$ be the continuous additive functional of $X$ with Revuz measure $\mu$ and let $g(x):=\mathbf{E}_{x}\left[e_{A+F}(\zeta)\right]$ be the gauge function. If $(X, A+F)$ is gaugeable, then for any $\delta>0$ there is a constant $c(\delta)>0$ such that

$$
\|g\|_{\infty}^{-1} g(x) \leq \sum_{n=0}^{\infty} \mathbf{E}_{x}\left[e_{A+F}\left(\tau_{n \delta}\right) ; \tau_{n \delta}<\zeta\right] \leq c(\delta)<\infty \quad \text { for all } x \in E .
$$

Proof. This lemma can be proved along the same line of argument as in Lemma 2.7 with measure $\mu^{+}+\mu_{F^{+}}$, the additive functionals $A+A^{F}$ and $A^{+}+A^{F^{+}}$in the roles of the measure $\mu^{+}$, and the additive functionals $A$ and $A^{+}$there respectively. The only places that need modifications are the estimates for $\mathbf{E}_{x}\left[e_{p A^{+}+p F^{+}}\left(\sigma_{K}\right)\right]$, $\mathbf{E}_{x}\left[e_{A^{+}+F^{+}}\left(\sigma_{K}\right)\right]$ and $\mathbf{E}_{x}\left[e_{A^{+}+F^{+}}\left(\tau_{j}\right) ; \tau_{j}<\zeta\right]$, which we give as follows.

Let $p>1$ be such that $p \mu^{+} \in \mathbf{K}_{1}(X)$ and define $F_{1}(x, y)=e^{p F^{+}(x, y)}-1$. Since $F^{+}$is bounded, we have

$$
F_{1} \leq p e^{\left\|p F^{+}\right\|_{\infty}} F^{+} \quad \text { and } \quad \mu_{F_{1}} \leq p e^{\left\|p F^{+}\right\|_{\infty}} \mu_{F^{+}} .
$$

With $A_{t}^{F_{1}}:=\sum_{0<s \leq t} F_{1}\left(X_{s-}, X_{s}\right)$,

$$
\begin{aligned}
e_{p A^{+}+p F^{+}}(t) & =\exp \left(p A_{t}^{+}+p \sum_{0<s \leq t} F^{+}\left(X_{s-}, X_{s}\right)\right) \\
& =\exp \left(p A_{t}^{+}\right) \prod_{0<s \leq t}\left(1+F_{1}\left(X_{s-}, X_{s}\right)\right) \\
& =\operatorname{Exp}\left(p A+A^{F_{1}}\right)_{t} .
\end{aligned}
$$

On the other hand, since

$$
\mathbf{E}_{x}\left[A_{t}^{F_{1}}\right]=\mathbf{E}_{x}\left[\int_{0}^{t} N F_{1}\left(X_{s}\right) d H_{s}\right] \leq \mathbf{E}_{x}\left[\int_{0}^{\infty} N F_{1}\left(X_{s}\right) d H_{s}\right]=U \mu_{F_{1}}(x),
$$

$\mathbf{E}_{x}\left[A_{\zeta}^{F_{1}}\right] \leq U \mu_{F_{1}}(x)<\infty$. So $A_{t}^{F_{1}}-\int_{0}^{t} N F_{1}\left(X_{s}\right) d H_{s}$ is a uniformly integrable martingale under every $\mathbf{P}_{x}$. Thus $\mathbf{E}_{x}\left[A_{\sigma_{K}}^{F_{1}}\right]=\mathbf{E}_{x}\left[\int_{0}^{\sigma_{K}} N F_{1}(X) d H_{s}\right] \leq U\left(1_{K^{c}} \mu_{F_{1}}\right)(x)$ and

$$
\begin{aligned}
\sup _{x \in E} \mathbf{E}_{x}\left[p A_{\sigma_{K}}^{+}+A_{\sigma_{K}}^{F_{1}}\right] & \leq\left\|U\left(1_{K^{c}}\left(p \mu^{+}+\mu_{F_{1}}\right)\right)\right\|_{\infty} \\
& \leq p\left\|U\left(1_{K^{c}}\left(\mu^{+}+e^{p\left\|F^{+}\right\|_{\infty}} \mu_{F^{+}}\right)\right)\right\|_{\infty} .
\end{aligned}
$$

Since $\nu:=p \mu^{+}+p e^{p\left\|F^{+}\right\|_{\infty}} \mu_{F^{+}} \in \mathbf{K}_{1}(X)$, in the display of (2.16) we can take a Borel set $K_{1}$ of finite $\nu$-mass and a constant $\delta>0$ so that

$$
p \sup _{B \subset K_{1}: \nu(B)<\delta}\left\|U\left(1_{K_{1}^{c} \cup B}\left(\mu^{+}+e^{2 \| F^{+}} \|_{\infty} \mu_{F^{+}}\right)\right)\right\|_{\infty}:=\beta_{1}<1 .
$$


Since $(X, A+F)$ is gaugeable, $g(x):=\mathbf{E}_{x}\left[e_{A+F}(\zeta)\right]$ is bounded and so

$$
\lim _{n \rightarrow \infty} \mathbf{E}_{x}\left[e_{A+F}(\zeta) ; \tau_{n}<\zeta\right]=0 \quad \text { on } E .
$$

Thus for any given small $\varepsilon>0$, one can find an integer $N$ large enough and a closed subset $K \subset K_{1}$ such that

$$
\sup _{x \in K} \mathbf{E}_{x}\left[e_{A+F}(\zeta) ; \tau_{N}<\zeta\right]<\varepsilon
$$

and $K_{1} \backslash K$ has mass less than $\delta$ under the measure $\mu^{+}+e^{2 \| F^{+}} \|_{\infty} \mu_{F^{+}}$. The set $K \subset K_{1}$ has the property that

$$
p\left\|U\left(1_{K^{c}}\left(\mu^{+}+e^{p\left\|F^{+}\right\|_{\infty}} \mu_{F^{+}}\right)\right)\right\|_{\infty} \leq \beta_{1}<1
$$

and so by Khasminskii's inequality (2.28),

$$
\sup _{x \in E} \mathbf{E}_{x}\left[e_{p A^{+}+p F^{+}}\left(\sigma_{K}\right)\right]=\sup _{x \in E} \mathbf{E}_{x}\left[\operatorname{Exp}\left(p A^{+}+A^{F_{1}}\right)_{\sigma_{K}}\right] \leq\left(1-\beta_{1}\right)^{-1} .
$$

Obviously,

$$
\sup _{x \in E} \mathbf{E}_{x}\left[e_{A^{+}+F^{+}}\left(\sigma_{K}\right)\right] \leq \sup _{x \in E} \mathbf{E}_{x}\left[e_{p A^{+}+p F^{+}}\left(\sigma_{K}\right)\right] \leq\left(1-\beta_{1}\right)^{-1} .
$$

To estimate $\mathbf{E}_{x}\left[e_{A^{+}+F^{+}}\left(\tau_{j}\right) ; \tau_{j}<\zeta\right]$, we use the Cauchy-Schwartz inequality:

$$
\begin{aligned}
\mathbf{E}_{x}[ & \left.e_{A^{+}+F^{+}}\left(\tau_{j}\right) ; \tau_{j}<\zeta\right] \\
\leq & \left(\mathbf{E}_{x}\left[\exp \left(2 A_{\tau_{j}}^{+}+2 \int_{0}^{\tau_{j}} N F^{+}\left(X_{s}\right) d H_{s}\right) ; \tau_{j}<\zeta\right]\right)^{1 / 2} \\
& \times\left(\mathbf{E}_{x}\left[\exp \left(2 \sum_{0<s \leq \tau_{j}} F^{+}\left(X_{s-}, X_{s}\right)-2 \int_{0}^{\tau_{j}} N F^{+}\left(X_{s}\right) d H_{s}\right)\right]\right)^{1 / 2} \\
\leq & 2^{j} .
\end{aligned}
$$

In the last inequality we used the definition of $\tau_{j}$ and the fact that

$$
t \mapsto \exp \left(2 \sum_{0<s \leq t} F^{+}\left(X_{s-}, X_{s}\right)-2 \int_{0}^{t} N F^{+}\left(X_{s}\right) d H_{s}\right)
$$

is a positive local martingale and therefore it is a supermartingale under $\mathbf{P}_{x}$. With these modifications, the proof for Lemma 2.7 carries over to get the conclusion of this lemma.

Theorem 2.15. Suppose that $\mu$ is a signed smooth measure in $\mathbf{K}_{1}(X)$ and $F \in$ $\mathbf{J}_{\infty}(X)$. Let $A^{+}$and $A^{-}$be the PCAFs with Revuz measures $\mu^{+}$and $\mu^{-}$respectively and $A=A^{+}-A^{-}$. If $(X, A+F)$ is gaugeable, then

$$
\sup _{x \in E} \mathbf{E}_{x}\left[\int_{0}^{\zeta} e_{A+F}(t) d\left(A_{t}^{+}+A_{t}^{F^{+}}\right)\right]<\infty
$$

Proof. Let $F_{1}:=e^{F^{+}}-1$. Since $F^{+}$is bounded, so is $F_{1}$ and therefore there is a constant $c_{1} \geq 1$ such that

$$
c^{-1} F_{1} \leq F^{+} \leq c F_{1}
$$


Note that

$$
\begin{aligned}
\int_{0}^{t} e_{A+F}(s) d\left(A_{s}^{+}+A_{s}^{F^{+}}\right) & \leq \int_{0}^{t} e_{A^{+}+F^{+}}(s) d\left(A_{s}^{+}+A_{s}^{F^{+}}\right) \\
& \leq c_{1} \int_{0}^{t} \operatorname{Exp}\left(A^{+}+A^{F_{1}}\right)_{s-} e^{F\left(X_{s-}, X_{s}\right)} d\left(A_{s}^{+}+A_{s}^{F_{1}}\right) \\
& \leq c_{1} e^{\|F\|_{\infty}}\left(\operatorname{Exp}\left(A^{+}+A^{F_{1}}\right)_{t}-1\right) .
\end{aligned}
$$

By the definition of $\tau_{t}$, Khasminskii's inequality (2.28) and the Markov property of $X$, we have

$$
C(t):=c_{1} e^{\left\|F_{1}\right\|_{\infty}} \sup _{x \in E} \mathbf{E}_{x}\left[\operatorname{Exp}\left(A^{+}+A^{F_{1}}\right)_{\tau_{t}}\right]<\infty .
$$

Now

$$
\begin{aligned}
\mathbf{E}_{x}\left[\int_{0}^{\zeta} e_{A+F}(t) d\left(A_{t}^{+}+A_{t}^{F^{+}}\right)\right] \\
\quad=\mathbf{E}_{x}\left[\sum_{n=0}^{\infty} \int_{\tau_{n} \wedge \zeta}^{\tau_{n+1} \wedge \zeta} e_{A+F}(t) d\left(A_{t}^{+}+A_{t}^{F^{+}}\right)\right] \\
\quad=\sum_{n=0}^{\infty} \mathbf{E}_{x}\left[\int_{\tau_{n}}^{\tau_{n+1} \wedge \zeta} e_{A+F}(t) d\left(A_{t}^{+}+A_{t}^{F^{+}}\right) ; \tau_{n}<\zeta\right] \\
=\sum_{n=0}^{\infty} \mathbf{E}_{x}\left[e_{A+F}\left(\tau_{n}\right) \mathbf{E}_{X_{\tau_{n}}}\left(\int_{0}^{\tau_{1} \wedge \zeta} e_{A+F}(t) d\left(A_{t}^{+}+A_{t}^{F^{+}}\right)\right) ; \tau_{n}<\zeta\right] \\
\leq C(1) \sum_{n=}^{\infty} \mathbf{E}_{x}\left[e_{A+F}\left(\tau_{n}\right): \tau_{n}<\zeta\right],
\end{aligned}
$$

which is bounded on $E$ by Lemma 2.14,

Remark 2.6. The above arguments can be easily modified, with $\tau_{t}$ being defined to be the right continuous inverse of the PCAF corresponding to the smooth measure $|\mu|+\mu_{|F|}$ as opposed to $\mu^{+}+\mu_{F^{+}}$, to show that if $\mu$ is a signed measure in $\mathbf{K}_{1}(X)$ and $F \in \mathbf{J}_{\infty}(X)$ such that $(X, \mu)$ is gaugeable, then

$$
\sup _{x \in E} \mathbf{E}_{x}\left[\int_{0}^{\zeta} e_{A+F}(t) d\left(|A|_{t}+A_{t}^{|F|}\right)\right]<\infty,
$$

where $|A|=A^{+}+A^{-}$is the PCAF corresponding to the Revuz measure $|\mu|=$ $\mu^{+}+\mu^{-}$.

Corollary 2.16. Suppose that $\mu$ is a signed smooth measure in $\mathbf{K}_{1}(X)$ and $F \in$ $\mathbf{J}_{\infty}(X)$. Let $A^{+}$and $A^{-}$be the PCAFs of $X$ with Revuz measures $\mu^{+}$and $\mu^{-}$ respectively, and let $A=A^{+}-A^{-}$. Then the following are equivalent:

(1) $(X, A+F)$ is gaugeable;

(2) $\mathbf{E}_{x}\left[\int_{0}^{\zeta} e_{A+F}(t) d\left(A_{t}^{+}+A_{t}^{F^{+}}\right)\right]<\infty$ for some $x \in E$;

(3) $\sup _{x \in E} \mathbf{E}_{x}\left[\int_{0}^{\zeta} e_{A+F}(t) d\left(A_{t}^{+}+A_{t}^{F^{+}}\right)\right]<\infty$;

(4) $\mathbf{E}_{x}\left[\sup _{t \leq \zeta} e_{A+F}(t)\right]<\infty$ for some $x \in E$;

(5) $\sup _{x \in E} \mathbf{E}_{x}\left[\sup _{t \leq \zeta} e_{A+F}(t)\right]<\infty$. 
Proof. Let $F_{1}=e^{F^{+}}-1$. It follows from (2.29) that

$$
\begin{aligned}
\int_{0}^{\zeta} & e_{A+F}(t) d\left(A_{t}^{+}+A_{t}^{F^{+}}\right) \\
& \geq e^{-\left(A_{\zeta}^{-}+A_{\zeta}^{F^{-}}\right)} \int_{0}^{\zeta} e_{A^{+}+F^{+}}(t-) e^{F^{+}\left(X_{t-}, X_{t}\right)} d\left(A_{t}^{+}+A_{t}^{F^{+}}\right) \\
& \geq c_{1}^{-1} e^{-\left(A_{\zeta}^{-}+A_{\zeta}^{F^{-}}\right)} \int_{0}^{\zeta} \operatorname{Exp}\left(A^{+}+F_{1}\right)_{t-} d\left(A_{t}^{+}+A_{t}^{F_{1}}\right) \\
& =c_{1}^{-1} e^{-\left(A_{\zeta}^{-}+A_{\zeta}^{F^{-}}\right)}\left(e^{A_{\zeta}^{+}+A_{\zeta}^{F^{+}}}-1\right) \\
& \geq c_{1}^{-1}\left(e_{A+F}(\zeta)-1\right) .
\end{aligned}
$$

The equivalence between (1)-(3) follows immediately from Theorems 2.13 and 2.15 For the remaining, observe that

$$
\begin{aligned}
e_{A+F}(t)=1 & +\int_{0}^{t} e_{A+F}(s-) d\left(A_{s}+A_{s}^{F}\right) \\
& +\sum_{0<s \leq t}\left(e_{A+F}(s)-e_{A+F}(s-)-e_{A+F}(s-) F\left(X_{s-}, X_{s}\right)\right) \\
=1 & +\int_{0}^{t} e_{A+F}(s-) d\left(A_{s}+A_{s}^{F^{+}}\right) \\
& +\sum_{0<s \leq t}\left(e_{A+F}(s)-e_{A+F}(s-)-e_{A+F}(s-) F^{+}\left(X_{s-}, X_{s}\right)\right) \\
\leq 1 & +c_{2} \int_{0}^{t} e_{A+F}(s-) d\left(A_{s}^{+}+A_{s}^{F^{+}}\right) \\
\leq 1 & +c_{2} e^{\|F\|_{\infty}} \int_{0}^{t} e_{A+F}(s) d\left(A_{s}^{+}+A_{s}^{F^{+}}\right) .
\end{aligned}
$$

In the second to last inequality, we used the fact that $F$ is bounded and so

$$
e_{A+F}(s)-e_{A+F}(s-)-e_{A+F}(s-) F^{+}\left(X_{s-}, X_{s}\right) \leq c_{3} e_{A+F}(s-) F^{+}\left(X_{s-}, X_{s}\right) .
$$

Therefore,

$$
e_{A+F}(\zeta) \leq \sup _{0 \leq t \leq \zeta} e_{A+F}(t) \leq 1+c_{2} \int_{0}^{\zeta} e_{A+F}(s) d\left(A_{s}^{+}+A_{s}^{F^{+}}\right)
$$

and the equivalence between (1)-(5) now follows.

By modifying the approach in the second proof of Theorem 7 in Chung and Rao [12], we can establish the following.

Theorem 2.17 (Super Gauge Theorem). Suppose that $\mu$ is a signed smooth measure in $\mathbf{K}_{1}(X), F \in \mathbf{J}_{\infty}(X)$ and that $\left(X, A^{\mu}+F\right)$ is gaugeable. Then there is an $\varepsilon_{0}>0$ such that $\left(X, A^{\mu+\varepsilon_{0}|\mu|}+F+\varepsilon_{0}|F|\right)$ is gaugeable. In particular, $\left(X, A^{(1+\varepsilon) \mu}+(1+\varepsilon) F\right)$ is gaugeable for all $\varepsilon \in\left[0, \varepsilon_{0}\right]$.

Proof. Let $A$ and $|A|$ be the continuous additive functionals of $X$ with Revuz measures $\mu$ and $|\mu|$ respectively and let $g(x):=\mathbf{E}_{x}\left[e_{A+F}(\zeta)\right]$ be the gauge function. 
Note that by (2.24), the function $g$ is bounded from below by a positive constant. We define a family of probability measures $\left\{\mathbf{Q}_{x}, x \in E\right\}$ by

$$
\mathbf{Q}_{x}(A)=g(x)^{-1} \mathbf{E}_{x}\left[e_{A+F}(\zeta) 1_{A}\right] \quad \text { for } A \in \mathcal{M}_{\infty} .
$$

To simplify notation we will also use $\mathbf{Q}_{x}$ to denote the expectation under probability measure $\mathbf{Q}_{x}$. For any $\left\{\mathcal{M}_{t}\right\}_{t \geq 0}$-stopping time $T$, by the strong Markov property of $X$, on $\{T<\zeta\}$,

$$
\begin{aligned}
\mathbf{Q}_{x}[ & \left.\left(|A|_{\zeta}+A_{\zeta}^{|F|}\right)-\left(|A|_{T-}+A_{T-}^{|F|}\right) \mid \mathcal{M}_{T}\right] \\
& \leq\|F\|_{\infty}+\mathbf{Q}_{x}\left[\left(|A|_{\zeta}+A_{\zeta}^{|F|}\right) \circ \theta_{T} \mid \mathcal{M}_{T}\right] \\
& =\|F\|_{\infty}+g\left(X_{T}\right)^{-1} \mathbf{E}_{x}\left[\left(e_{A+F}(\zeta)\left(|A|_{\zeta}+A_{\zeta}^{|F|}\right)\right) \circ \theta_{T} \mid \mathcal{M}_{T}\right] \\
& =\|F\|_{\infty}+g\left(X_{T}\right)^{-1} \mathbf{E}_{X_{T}}\left[e_{A+F}(\zeta)\left(|A|_{\zeta}+A_{\zeta}^{|F|}\right)\right] .
\end{aligned}
$$

On the other hand, by Remark [2.6,

$$
\begin{aligned}
\sup _{x \in E} & \mathbf{E}_{x}\left[e_{A+F}(\zeta)\left(|A|_{\zeta}+A_{\zeta}^{|F|}\right)\right] \\
& =\sup _{x \in E} \mathbf{E}_{x}\left[\int_{0}^{\zeta} e_{A+F}(\zeta) d\left(|A|_{t}+A_{t}^{|F|}\right)\right] \\
& =\sup _{x \in E} \mathbf{E}_{x}\left[\int_{0}^{\zeta} e_{A+F}(t) \mathbf{E}_{X_{t}}\left[e_{A+F}(\zeta)\right] d\left(|A|_{t}+A_{t}^{|F|}\right)\right] \\
& \leq\|g\|_{\infty} \sup _{x \in E} \mathbf{E}_{x}\left[\int_{0}^{\zeta} e_{A+F}(t) d\left(|A|_{t}+A_{t}^{|F|}\right)\right] \\
:= & c_{1}<\infty .
\end{aligned}
$$

Hence

$$
\begin{aligned}
& \mathbf{Q}_{x}\left[\left(|A|_{\zeta}+A_{\zeta}^{|F|}\right)-\left(|A|_{T-}+A_{T-}^{|F|}\right) \mid \mathcal{M}_{T}\right] \\
& \quad \leq\|F\|_{\infty}+c_{1}\left\|g^{-1}\right\|_{\infty} .
\end{aligned}
$$

Now replacing $A$ and $F$ above by $\varepsilon_{0} A$ and $\varepsilon_{0} F$, for $\varepsilon_{0}<c_{2}^{-1}$ we have

$$
\mathbf{Q}_{x}\left[\left(\varepsilon_{0}|A|_{\zeta}+A_{\zeta}^{\varepsilon_{0}|F|}\right)-\left(\varepsilon_{0}|A|_{T-}+A_{T-}^{\varepsilon_{0}|F|}\right) \mid \mathcal{M}_{T}\right] \leq \theta<1 \quad \text { for every } x \in E .
$$

Hence by John-Nirenberg's inequality (see VI.190 of Dellacherie and Meyer [15]),

$$
\sup _{x \in E} \mathbf{Q}_{x}\left[e_{\varepsilon_{0}|A|+\varepsilon_{0}|F|}(\zeta)\right]<\infty
$$

and therefore

$$
\begin{aligned}
\sup _{x \in E} \mathbf{E}_{x}\left[e_{\left(A+\varepsilon_{0}|A|\right)+\left(F+\varepsilon_{0}|F|\right)}(\zeta)\right] & =\sup _{x \in E} \mathbf{E}_{x}\left[e_{A+F}(\zeta) e_{\varepsilon_{0}|A|+\varepsilon_{0}|F|}(\zeta)\right] \\
& =\sup _{x \in E}\left(g(x) \mathbf{Q}_{x}\left[e_{\varepsilon_{0}|A|+\varepsilon_{0}|F|}(\zeta)\right]\right)<\infty .
\end{aligned}
$$

So $\left(X,\left(A+\varepsilon_{0}|A|\right)+\left(F+\varepsilon_{0}|F|\right)\right)$ is gaugeable. Since

$$
(1+\varepsilon) A_{t}+A_{t}^{(1+\varepsilon) F} \leq A_{t}+\varepsilon_{0}|A|_{t}+A_{t}^{F+\varepsilon_{0}|F|} \text { for } \varepsilon \in\left[0, \varepsilon_{0}\right],
$$

$\left(X, A^{(1+\varepsilon) \mu}+(1+\varepsilon) F\right)$ is gaugeable for all $\varepsilon \in\left[0, \varepsilon_{0}\right]$. 
Remark 2.7. When $X$ is symmetric, Theorems 2.11and2.12 can also be established for $\left(X, A^{\mu}+F\right)$ and the Schrödinger semigroup

$$
Q_{t} f(x)=\mathbf{E}_{x}\left[e_{A^{\mu}+F}(t) f\left(X_{t}\right)\right]
$$

with almost the same proofs modulo the modifications as those made in the proofs for Lemma 2.14 up to Corollary 2.16, where $\mu \in \mathbf{K}_{1}(X)$ and $F \in \mathbf{J}_{\infty}(X)$ is symmetric. So we omit the details here.

\section{Conditional Gauge theorems}

In addition to the assumptions on $X$ made at the beginning of the last section, suppose now that there is another transient Borel right process

$$
\widehat{X}=\left(\widehat{\Omega}, \widehat{\mathcal{M}}, \widehat{\mathcal{M}}_{t}, \widehat{X}_{t}, \widehat{\mathbf{P}}_{x}, x \in E\right)
$$

on the same state space $E$ which is a strong dual of $X$ with respect to the measure $m$. That is, the semigroup $\left\{\widehat{P}_{t}\right\}_{t \geq 0}$ of $\widehat{X}$ is the dual in $L^{2}(E, m)$ to the semigroup $\left\{P_{t}\right\}_{t \geq 0}$ of $X$ :

$$
\int_{E} f(x) P_{t} g(x) m(d x)=\int_{E} g(x) \widehat{P}_{t} f(x) m(d x) \quad \text { for all } f, g \in L^{2}(E, m)
$$

and the resolvents $\left\{U_{\alpha}\right\}$ and $\left\{\widehat{U}_{\alpha}\right\}$ satisfy the following conditions: for each $\alpha>0$, a $(\mathcal{B}(E) \times \mathcal{B}(E))$-measurable potential density $G_{\alpha}(x, y)$ can be chosen so that

(a) $\quad U_{\alpha}(x, d y)=G_{\alpha}(x, y) m(d y), \quad \widehat{U}_{\alpha}(x, d y)=G_{\alpha}(y, x) m(d y)$

(b) $x \rightarrow G_{\alpha}(x, y)$ is $\alpha$-excessive for $X, \quad y \rightarrow G_{\alpha}(x, y)$ is $\alpha$-excessive for $\widehat{X}$.

When $\alpha=0$, we will drop the subscript and write $G$ for $G_{0}$. In this setting we do not need to assume a priori that the Borel right process $X$ has left limits in $(0, \zeta)$. The strong duality assumption above implies that both $X$ and $\widehat{X}$ have left limits on $(0, \zeta)$ and $(0, \widehat{\zeta})$ respectively; more precisely, $X_{t-}$ and $\widehat{X}_{t-}$ exist in $E$ for all $t \in(0, \zeta)$ and $t \in(0, \widehat{\zeta})$ respectively. Note that under the above duality assumption, the Revuz formula (2.2) holds and therefore for any positive smooth measure $\mu, U \mu=G \mu=\int_{E} G(\cdot, y) \mu(d y)$.

The above assumptions also imply that an excessive function $h$ of $X$ is Borel measurable. For any excessive function $h$ of $X$ that is finite $m$-a.e. on $E$, let $E_{h}:=\{x \in E: 0<h(x)<\infty\}$ and define

$$
p^{h}(t, x, d y)=\frac{h(y) p(t, x, d y)}{h(x)}, \quad t>0, x, y \in E_{h} .
$$

Then $p^{h}$ is a transition probability and determines a Borel right process $X^{h}$ on $E_{h}$ (cf. Getoor and Glover [25]), which is called Doob's $h$-transformed process of $X$. Let $E \backslash E_{h}$ be absorbing states for $X^{h}$. Then by Proposition 5.4 of [25], $X^{h}$ and $\widehat{X}$ are in strong dual on $E$ with respect to the measure $h m$ and therefore $X^{h}$ has left limits on $\left(0, \zeta^{h}\right)$. Note that $m\left(E \backslash E_{h}\right)=0$. We are going to use $\zeta^{h}$ to denote the lifetime of the $h$-conditioned process. For any $x \in E$, we are going to use $\mathbf{P}_{x}^{h}$ and $\mathbf{E}_{x}^{h}$ to denote the probability and expectation with respect to the $h$-conditioned process starting from $x$ respectively. When $h(\cdot)=G(\cdot, y)$ for some $y \in E$, we will use $\mathbf{P}_{x}^{y}$ and $\mathbf{E}_{x}^{y}$ to denote the probability and expectation for the $h$-conditioned process starting from $x$ respectively. In this case, the lifetime $\zeta^{h}$ will be denoted as $\zeta^{y}$ and the process $X^{h}$ as $X^{y}$. 
It is clear that if $u$ is an excessive function for $X^{h}$, then $u h$ is excessive for $X$. Furthermore, if $A^{\mu}$ is a PCAF of $X$ with Revuz measure $\mu$, then by Proposition 5.1 of Getoor and Glover [25] and (2.1)

$$
\begin{aligned}
\lim _{t \downarrow 0} \mathbf{E}_{h m}^{h}\left[\frac{1}{t} \int_{0}^{t} f\left(X_{s}^{h}\right) d A_{s}^{\mu}\right] & =\lim _{t \downarrow 0} \mathbf{E}_{m}\left[\frac{1}{t} \int_{0}^{t}(h f)\left(X_{s}\right) d A_{s}\right] \\
& =\int_{E} f(x) h(x) \mu(d x),
\end{aligned}
$$

for positive $f \geq 0$ on $E_{h}$. Here we used the fact that $h m$ is an excessive measure for $X^{h}$. The above implies that $A^{\mu}$ is a PCAF of $X^{h}$ with Revuz measure $h(x) \mu(d x)$.

Note that by (3.1), $X^{h}$ has Green function

$$
G^{h}(x, y):=\frac{G(x, y)}{h(x)}
$$

with respect to measure $h m$.

3.1. Conditional Gauge Theorem for Local Perturbations. The following definitions for Kato classes $\mathbf{S}_{\infty}(X)$ and $\mathbf{S}_{1}(X)$ are extensions of the corresponding classes in Chen and Song 8 to measures that may not be absolutely continuous with respect to $m$. Define $d:=\{(x, w) \in E \times E: G(x, w)=0$ or $\infty\}, E_{w}:=\{x \in$ $E: 0<G(x, w)<\infty\}$ and $\widehat{E}_{w}:=\{x \in E: 0<G(w, x)<\infty\}$.

Definition 3.1. (1) A signed smooth measure is said to be in the class $\mathbf{S}_{\infty}(X)$ if for any $\varepsilon>0$ there is a Borel subset $K=K(\varepsilon)$ of finite $|\mu|$-measure and a constant $\delta=\delta(\varepsilon)>0$ such that

$$
\sup _{(x, z) \in(E \times E) \backslash d} \int_{K^{c}} \frac{G(x, y) G(y, z)}{G(x, z)}|\mu|(d y) \leq \varepsilon
$$

and for all measurable sets $B \subset K$ with $|\mu|(B)<\delta$,

$$
\sup _{(x, z) \in(E \times E) \backslash d} \int_{B} \frac{G(x, y) G(y, z)}{G(x, z)}|\mu|(d y) \leq \varepsilon .
$$

(2) A signed smooth measure $\mu$ is said to be in class $\mathbf{S}_{1}(X)$ if there is a Borel set $K$ of finite $|\mu|$-measure and a constant $\delta>0$ such that

$$
\beta_{2}(\mu):=\sup _{B \subset K:|\mu|(B)<\delta} \sup _{(x, z) \in(E \times E) \backslash d} \int_{K^{c} \cup B} \frac{G(x, y) G(y, z)}{G(x, z)}|\mu|(d y)<1 .
$$

By the same proof as that for Corollary 3.1 in Chen and Song [8], we have $\mathbf{S}_{\infty}(X) \subset \mathbf{K}_{\infty}(X)$ and $\mathbf{S}_{1}(X) \subset \mathbf{K}_{1}(X)$.

When $X$ is a symmetric $\alpha$-stable process in $\mathbf{R}^{n}$ with $n>\alpha$, it is well known that its Green function $G(x, y)=c(n, \alpha)|x-y|^{\alpha-n}$ and the following $3 G$-inequality holds:

$$
\frac{G(x, y) G(y, z)}{G(x, z)} \leq C(G(x, y)+G(y, z)) .
$$

So $\mathbf{S}_{\infty}(X) \supset \mathbf{K}_{\infty}(X)$. We can also consider the Kato class $\mathbf{S}_{\infty}$ for the subprocess of $X$. For domain $D \subset \mathbf{R}^{n}$, let $X^{D}$ be the subprocess of $X$ killed upon leaving $D$ 
and let $G_{D}$ denote its Green function. When $D$ is a bounded $C^{1,1}$-smooth domain, it is known (see Chen and Song [6] and Kulczycki [26]) that

$$
G_{D}(x, y) \approx \min \left\{\frac{1}{|x-y|^{n-\alpha}}, \frac{\delta_{D}(x)^{\alpha / 2} \delta_{D}(y)^{\alpha / 2}}{|x-y|^{n-\alpha}}\right\} \quad \text { for } x, y \in D .
$$

Here $\delta_{D}(x)$ is the Euclidean distance between $x$ and $D^{c}$, and $f \approx g$ means there are $c_{1}, c_{2}>0$ such that $c_{1} g \leq f \leq c_{2} g$. We will not delve too far into concrete examples of $\mathbf{S}_{\infty}\left(X^{D}\right)$-measures but will only mention the following.

Proposition 3.1. (1) A positive measure $\mu$ is in $\mathbf{S}_{\infty}\left(X^{D}\right)$ if and only if for every relatively compact open set $U \subset D, 1_{U} \mu \in \mathbf{K}(X)$ and that for any $\varepsilon>0$, there is a compact subset $K$ of $D$ such that

$$
\sup _{x, y \in D} \frac{1}{G_{D}(x, y)} \int_{D \backslash K} G_{D}(x, z) G_{D}(z, y) \mu(d z) \leq \varepsilon .
$$

(2) Suppose that $D$ is a bounded $C^{1,1}$-domain in $\mathbf{R}^{n}$. Then a positive measure $\mu$ is in $\mathbf{S}_{\infty}\left(X^{D}\right)$ if and only if for every relatively compact open set $U \subset D, 1_{U} \mu \in$ $\mathbf{K}(X)$ and that for any $\varepsilon>0$ there is a compact subset $K=K(\varepsilon)$ of $D$ such that

$$
\sup _{x \in D \backslash K} \int_{D \backslash K} \frac{\delta_{D}(y)^{\alpha / 2}}{\delta_{D}(x)^{\alpha / 2}} G_{D}(x, y) \mu(d y) \leq \varepsilon .
$$

Proof. The proof is similar to those for Propositions 4.1 and 4.2 in Chen and Song 8], combined with applying Theorem 1 of Zhao [4] on a relatively compact smooth subdomain $U$ of $D$. So we omit the details here.

Using estimate (3.6) and the characterization in Proposition [3.1 2 , concrete conditions (see [8] ) can be given for $\mu \in \mathbf{S}_{\infty}\left(X^{D}\right)$.

Proposition 3.2. For positive measure $\mu \in \mathbf{S}_{1}(X)$,

$$
\sup _{(x, w) \in(E \times E) \backslash d} \int_{E_{w}} \frac{G(x, y) G(y, z)}{G(x, w)} \mu(d y)<\infty .
$$

Proof. The proof is similar to that for Proposition 2.2 so we omit it here.

Suppose that $\mu$ is a smooth measure of $X$ with $\mu^{+} \in \mathbf{S}_{1}(X)$. Let $A^{\mu,+}$ and $A^{\mu,-}$ be the PCAFs of $X$ with Revuz measures $\mu^{+}$and $\mu^{-}$respectively. We know from (3.2) that $A^{\mu,+}$ and $A^{\mu,-}$ can be regarded as PCAFs of $X^{\cdot, w}$ with Revuz measures $G(\cdot, w) \mu^{+}$and $G(\cdot, w) \mu^{-}$respectively. Since $\mu^{+} \in \mathbf{S}_{1}(X)$, we have by Proposition 3.2 and (3.3) that $\sup _{x \in E_{w}} \mathbf{E}_{x}^{w}\left[A_{\zeta^{w}}^{\mu,+}\right]<\infty$ for every $w$. Thus the conditional gauge function $\mathbf{E}_{x}^{w}\left[\exp \left(A_{\zeta^{w}}^{\mu}\right)\right]$ is well defined for $(x, y) \notin d$.

Theorem 3.3 (Conditional Gauge Theorem I). Suppose that $\mu$ is a signed smooth measure of $X$ such that $\mu^{+} \in \mathbf{S}_{1}(X)$. If $\mathbf{E}_{x}^{w}\left[e_{A}\left(\zeta^{w}\right)\right]$ is finite for some $\left(x_{0}, w_{0}\right) \in$ $(E \times E) \backslash d$, then $\mathbf{E}_{x}^{w}\left[e_{A}\left(\zeta^{w}\right)\right]$ is bounded on $(E \times E) \backslash d$.

Proof. Applying the same idea as that in the proof of Theorem 2.5(2), it can be shown that $G(\cdot, w) \mu^{+} \in \mathbf{K}_{1}\left(X^{w}\right)$ for each $w \in E$. The rest of the proof is the same as that for Theorem 3.3 in Chen and Song [8], modulo some straightforward modifications. 
The conditional gauge theorem is important since the conditional gauge function is the ratio of the Green function $G_{\mathcal{L}+\mu}$ for the Schrödinger operator $\mathcal{L}+\mu$ with the Green function $G$ of $X$ (see Lemma 3.5 below). Here $\mathcal{L}$ is the infinitesimal generator of (the semigroup of) $X$ and $\mathcal{L}+\mu$ denotes the infinitesimal generator of the Schrödinger semigroup $Q_{t}$ defined by $Q_{t} f(x)=\mathbf{E}_{x}\left[e_{A}(t) f\left(X_{t}\right)\right]$. To the author's knowledge, this is the first time a conditional gauge theorem is explicitly established for a potential $\mu$ that is not absolutely continuous with respect to $m$, even when $X$ is a Brownian motion in $\mathbf{R}^{n}$ or in a domain $D \subset \mathbf{R}^{n}$.

Definition 3.2. For a signed smooth measure $\mu$ of $X$ with $\mu^{+} \in \mathbf{S}_{1}(X)$, we say that $(X, \mu)$ is conditionally gaugeable if the conditional gauge function $(x, w) \mapsto$ $\mathbf{E}_{x}^{w}\left[e_{A}\left(\zeta^{w}\right)\right]$ is bounded on $(E \times E) \backslash d$.

It is possible to have the analogy of Corollary 2.9 for conditional gaugeability.

Theorem 3.4. Suppose that $\mu$ is a signed smooth measure such that $\mu^{+} \in \mathbf{S}_{1}(X)$ and that

$$
\sup _{(x, w) \in E \times E) \backslash d} \int_{E_{w}} \frac{G(x, y) G(y, w)}{G(x, w)} \mu^{-}(d y)<\infty .
$$

Let $A^{+}$and $A^{-}$be the PCAFs of $X$ with Revuz measures $\mu^{+}$and $\mu^{-}$respectively. Then the following are equivalent:

(1) $(X, \mu)$ is conditionally gaugeable;

(2) $\mathbf{E}_{x}^{w}\left[\int_{0}^{\zeta^{w}} e_{A}(t) d A_{t}^{+}\right]<\infty$ for some $(x, w) \in(E \times E) \backslash d$;

(3) $\sup _{(x, w) \in(E \times E) \backslash d} \mathbf{E}_{x}^{w}\left[\int_{0}^{\zeta^{w}} e_{A}(t) d A_{t}^{+}\right]<\infty$;

(4) $\mathbf{E}_{x}^{w}\left[\sup _{t \leq \zeta^{w}} e_{A}(t)\right]<\infty$ for some $(x, w) \in(E \times E) \backslash d$;

$(5) \sup _{(x, w) \in(E \times E) \backslash d} \mathbf{E}_{x}^{w}\left[\sup _{t \leq \zeta^{w}} e_{A}(t)\right]<\infty$.

Proof. In view of (2.18), it suffices to show the equivalence between (1)-(3). For $(x, w) \in(E \times E) \backslash d$, define

$$
u(x, w)=\mathbf{E}_{x}^{w}\left[\int_{0}^{\zeta^{w}} e_{A}(t) d A_{t}^{+}\right] \text {and } \widehat{u}(x, w)=\widehat{\mathbf{E}}_{w}^{x}\left[\int_{0}^{\widehat{\zeta}^{w}} e_{A}(t) d A_{t}^{+}\right] .
$$

Suppose that (1) holds. Applying Corollary 2.9 to the conditional process $\left(\widehat{X}^{w}, \mathbf{P}_{x}^{w}, x \in \widehat{E}_{w}\right)$, where $\widehat{E}_{w}=\{x \in E: 0<G(w, x)<\infty\}$, we have $\sup _{x \in \widehat{E}_{w}} \widehat{u}(x, w)<\infty$ for any $w \in E$. By Theorem 6.5 and Example 6.14 of Getoor and Glover [25], for any $(x, w) \in(E \times E) \backslash d$, the time reversal at its lifetime of the process $\left(X, \mathbf{P}_{x}^{w}\right)$ (obtained by reversing the conditional process $\left(X^{w}, \mathbf{P}_{x}^{w}\right)$ at its lifetime $\zeta^{w}$ and taking the right continuous version) has the same distribution as the conditional process $\left(\widehat{X}^{x}, \widehat{\mathbf{P}}_{w}^{x}\right)$. Consequently, $u(x, w)=\widehat{u}(w, x)$ and so $\sup _{x \in \widehat{E}_{w}} u(w, x)<\infty$ for every $w \in E$. Let $K$ be the Borel set and $\delta$ the positive constant in Definition 3.1 for $\mu^{+} \in \mathbf{S}_{1}(X)$ such that

$$
\begin{aligned}
\sup _{(x, z) \in(E \times E) \backslash d}\left(\int_{E \backslash K} \frac{G(x, y) G(y, z)}{G(x, z)} \mu^{+}(d y)\right. \\
\left.\quad+\sup _{B \subset K:|\mu|(B)<\delta} \int_{B} \frac{G(x, y) G(y, z)}{G(x, z)} \mu^{+}(d y)\right):=\beta_{2}\left(\mu^{+}\right)<1 .
\end{aligned}
$$


Note that $u(x, w)$ is $\mathcal{B}(E \times E)$ if we set $u(x, w)=1$ for $(x, w) \in d$. Hence $\{x \in$ $\left.K: \sup _{w \in E} u(x, w)>M\right\}$ is $\mathcal{B}(E)$-measurable since it is the $x$-projection of the set $\{(x, w) \in K \times E: u(x, w)>M\}$. Since $\bigcap_{M=2}^{\infty}\left\{x \in K: \sup _{w \in E} u(x, w)>M\right\}=\emptyset$, we can choose $M$ large enough so that the set $\left\{x \in K: \sup _{w \in E} u(x, w)>M\right\}$ has $\mu^{+}$-measure less than $\delta / 2$. Choose a closed subset $K_{1}$ of $K$ such that $K \backslash K_{1} \supset$ $\left\{x \in K: \sup _{z \in E} u(x, w)>M\right\}$ and has $\mu^{+}$-mass less than $\delta$. Note that since $K_{1}^{c}=K^{c} \cup\left(K \backslash K_{1}\right)$,

$$
\sup _{(x, w) \in E \times E) \backslash d} \int_{K_{1}^{c}} \frac{G(x, y) G(y, w)}{G(x, w)} \mu^{+}(d y) \leq \beta_{2}\left(\mu^{+}\right)<1 .
$$

Now by the strong Markov property of $X^{w}$, for every $(x, w) \in(E \times E) \backslash d$,

$$
\begin{aligned}
u(x, w) & =\mathbf{E}_{x}^{w}\left[\int_{0}^{\sigma_{K_{1}}} e_{A}(t) d A_{t}^{+}\right]+\mathbf{E}_{x}^{w}\left[\int_{\sigma_{K_{1}}}^{\zeta^{w}} e_{A}(t) d A_{t}^{+} ; \sigma_{K_{1}}<\zeta^{w}\right] \\
& \leq \mathbf{E}_{x}^{w}\left[\int_{0}^{\sigma_{K_{1}}} e_{A^{+}}(t) d A_{t}^{+}\right]+\mathbf{E}_{x}^{w}\left[e_{A}\left(\sigma_{K_{1}}\right) u\left(X_{\sigma_{K_{1}}}^{w}, w\right) ; \sigma_{K_{1}}<\zeta^{w}\right] \\
& \leq(M+1) \mathbf{E}_{x}^{w}\left[\exp \left(A_{\sigma_{K_{1}}}^{+}\right)\right]-1 \\
& \leq \frac{M+1}{1-\beta_{2}\left(\mu^{+}\right)}-1 .
\end{aligned}
$$

In the second to last inequality, we used the fact that $K_{1}$ is closed and therefore $X_{\sigma_{K_{1}}}^{w} \in K_{1}$ and so $u\left(X_{\sigma_{K_{1}}}^{w}, w\right) \leq M$ on $\left\{\sigma_{K_{1}}<\zeta^{w}\right\}$; in the last inequality, we used (3.8) and Khasminskii's lemma. This proves that (3) follows from (1). That (3) implies (2) is trivial. Now suppose (2) holds for some $(x, w)$. Then by (2.17), $\mathbf{E}_{x}^{w}\left[e_{A}\left(\zeta^{w}\right)\right]<\infty$ and therefore by Theorem $3.3(X, \mu)$ is conditionally gaugeable.

Note that by Proposition 3.2, condition (3.7) is satisfied if $\mu$ is a signed measure in $\mathbf{S}_{1}(X)$.

Suppose that $\mu$ is a signed measure with $\mu^{+} \in \mathbf{K}_{1}(X)$ and $G \mu^{-}$being bounded. Let $A^{+}$and $A^{-}$be the PCAFs of $X$ with Revuz measures $\mu^{+}$and $\mu^{-}$respectively, and let $A=A^{+}-A^{-}$. Define the Schrödinger semigroup

$$
Q_{t} f(x):=\mathbf{E}_{x}\left[e_{A}(t) f\left(X_{t}\right) d t\right]
$$

and the resolvent

$$
V f(x):=\int_{0}^{\infty} Q_{t} f(x) d x=\mathbf{E}_{x}\left[\int_{0}^{\infty} e_{A}(t) f\left(X_{t}\right) d t\right] .
$$

Clearly $Q_{t}$ is a bounded semigroup in $L^{\infty}(E, m)$ and we formally denote the infinitesimal generator of $Q_{t}$ as $\mathcal{L}+\mu$. Note that the semigroup $Q_{t}$ can be viewed as the Feynman-Kac semigroup of $Y$ by the transformation $e^{A_{t}^{+}}$, where $Y$ is the subprocess of $X$ killed at rate $\mu^{-}$as mentioned above. When $\mu^{+} \in \mathbf{K}_{1}(X) \cap \mathbf{K}_{1}(\widehat{X})$, it follows from Proposition 2.3 above and Theorem 4.15 in Getoor [24] that the Schrödinger semigroup $Q_{t}$ is strongly continuous in $L^{p}(E, m)$ for every $1 \leq p<\infty$.

The following lemma gives the explicit expression for the Green function of $\mathcal{L}+\mu$. Its simple proof using the property of $h$-transform seems to be new even in the classical Brownian motion case (cf. [13] and [14]). 
Lemma 3.5. (1) For every Borel function $f \geq 0$,

$$
\mathbf{E}_{x}\left[\int_{0}^{\infty} e_{A}(t) f\left(X_{t}\right) d t\right]=\int_{E} G(x, y) \mathbf{E}_{x}^{y}\left[e_{A}\left(\zeta^{y}\right)\right] f(y) m(d y) .
$$

That is, $V(x, y):=G(x, y) \mathbf{E}_{x}^{y}\left[e_{A}\left(\zeta^{y}\right)\right]$ is the Green function of $\mathcal{L}+\mu$.

(2) For any positive measure $\nu \in \mathbf{K}_{1}(X)$,

$$
\mathbf{E}_{x}\left[\int_{0}^{\infty} e_{A}(t) d A_{t}^{\nu}\right]=\int_{E} G(x, y) \mathbf{E}_{x}^{y}\left[e_{A}\left(\zeta^{y}\right)\right] \nu(d y),
$$

where $A^{\nu}$ is the PCAF of $X$ with Revuz measure $\nu$.

Proof. (1) Fix a bounded Borel measurable function $f_{0} \in L^{2}(E, m)$ that is strictly positive $m$-a.e. on $E$ and such that $h(x):=\int G(x, y) f_{0}(y) m(d y)$ is bounded. The existence of such $f_{0}$ is guaranteed by Getoor [23] since $X$ is transient. Clearly $h$ is an excessive function of $X$ and so $h\left(X_{t}\right)$ is a supermartingale under $\mathbf{P}_{x}$ for every $x \in E$. Moreover,

$$
M_{t}=h\left(X_{t}\right)-h\left(X_{0}\right)+\int_{0}^{t} f_{0}\left(X_{s}\right) d s
$$

is an $L^{2}$-integrable martingale under $\mathbf{P}_{x}$ with

$$
\sup _{t \geq 0} \mathbf{E}_{x}\left[M_{t}^{2}\right]<\infty
$$

for every $x \in E$. The latter is because $h$ is bounded and that

$$
\begin{aligned}
\mathbf{E}_{x}\left[\left(\int_{0}^{\infty} f_{0}\left(X_{s}\right) d s\right)^{2}\right] & =2 \mathbf{E}_{x}\left[\int_{0}^{\infty} f_{0}\left(X_{s}\right) \mathbf{E}_{X_{s}}\left[\int_{0}^{\infty} f_{0}\left(X_{r}\right) d r\right] d s\right] \\
& \leq 2\left\|G f_{0}\right\|_{\infty}^{2}<\infty .
\end{aligned}
$$

By Proposition 5.1 of Getoor and Glover [25] and 62 of Sharpe [33],

$$
\begin{aligned}
\int_{E} G(x, y) \mathbf{E}_{x}^{y}\left[e_{A}\left(\zeta^{y}\right) \wedge n\right] f_{0}(y) m(d y)=h(x) \mathbf{E}_{x}^{h}\left[e_{A}\left(\zeta^{h}\right) \wedge n\right] \\
\quad=\mathbf{E}_{x}\left[\int_{0}^{\infty}\left(e_{A}(t) \wedge n\right)\left(-d M_{t}+f_{0}\left(X_{t}\right) d t\right)\right] \\
\quad=\mathbf{E}_{x}\left[\int_{0}^{\infty}\left(e_{A}(t) \wedge n\right) f_{0}\left(X_{t}\right) d t\right] .
\end{aligned}
$$

In the last identity we used the fact that $\mathbf{E}_{x}\left[\int_{0}^{\infty}\left(e_{A}(t) \wedge n\right) d M_{t}\right]=0$, which is a consequence of (3.11). Letting $n \rightarrow \infty$, we have

$$
\int_{E} G(x, y) \mathbf{E}_{x}^{y}\left[e_{A}\left(\zeta^{y}\right)\right] f_{0}(y) m(d y)=\mathbf{E}_{x}\left[\int_{0}^{\infty} e_{A}(t) f_{0}\left(X_{t}\right) d t\right] .
$$

For a general Borel $f \geq 0, f_{k}:=f \wedge\left(k f_{0}\right)$ is in $L^{2}(E, m)$ with $G f_{k}$ being bounded. So we have from above

$$
\mathbf{E}_{x}\left[\int_{0}^{\infty} e_{A}(t) f_{k}\left(X_{t}\right) d t\right]=\int_{E} G(x, y) \mathbf{E}_{x}^{y}\left[e_{A}\left(\zeta^{y}\right)\right] f_{k}(y) m(d y) .
$$

Passing $k \rightarrow \infty$ proves Part (1) of the lemma. 
(2) For a positive measure $\nu \in \mathbf{K}_{1}(X)$, by Proposition [2.2, function $x \mapsto$ $\mathbf{E}_{x}\left[A_{\zeta}^{\nu}\right]=G \nu(x)$ is bounded on $E$ and therefore by a similar argument to that for 3.12 we have

$$
\mathbf{E}_{x}\left[\left(A_{\zeta}^{\nu}\right)^{2}\right] \leq 2\|G \nu\|_{\infty}<\infty
$$

The rest of the proof is almost identical to that for (1) with $h(x):=G \nu(x)$ and $A^{\nu}$ in place of $\int_{0}^{t} f_{0}\left(X_{s}\right) d s$.

Now we can show the equivalence of gaugeability and conditional gaugeability of $(X, \mu)$, and relate them to the subcriticality of $\mathcal{L}+\mu$ (cf. Pinchover [30] and Zhao [43]).

Definition 3.3. We call the Schrödinger operator $\mathcal{L}+\mu$ subcritical if there is some $x_{0} \in E$ and a Borel subset $B \subset E$ of positive $m$-measure such that $V 1_{B}\left(x_{0}\right)<\infty$, where the resolvent $V$ is defined by (3.10).

Theorem 3.6. Suppose that $\mu$ is a signed smooth measure such that $\mu^{+} \in \mathbf{S}_{1}(X)$ and $\mu^{-}$satisfies (3.7). Then the following are equivalent:

(1) $(X, \mu)$ is gaugeable;

(2) $(X, \mu)$ is conditionally gaugeable;

(3) $\mathcal{L}+\mu$ is subcritical.

Proof. Note that $\mu^{+} \in \mathbf{S}_{1}(X) \subset \mathbf{K}_{1}(X)$. By the same argument as that for Corollary 3.1 in Chen and Song [8], condition (3.7) implies that $G \mu^{-}$is bounded. Let $A^{+}$and $A^{-}$be the PCAFs of $X$ with Revuz measures $\mu^{+}$and $\mu^{-}$respectively.

Suppose $(X, \mu)$ is gaugeable. Then by Lemma 3.5 (2) and Corollary 2.9

$$
\sup _{x \in E} \int_{E} G(x, y) \mathbf{E}_{x}^{y}\left[e_{A}\left(\zeta^{y}\right)\right] \mu^{+}(d y)=\sup _{x \in E} \mathbf{E}_{x}\left[\int_{0}^{\infty} e_{A}(t) d A_{t}^{+}\right]<\infty .
$$

Thus there is some $(x, y) \in(E \times E) \backslash d$ such that $\mathbf{E}_{x}^{y}\left[e_{A}\left(\zeta^{y}\right)\right]<\infty$ and so, by Theorem $3.3,(X, \mu)$ is conditionally gaugeable. Conversely, if $(X, \mu)$ is conditionally gaugeable, it follows from the same identity above that

$$
\sup _{x \in E} \mathbf{E}_{x}\left[\int_{0}^{\infty} e_{A}(t) d A_{t}^{+}\right] \leq c\left\|G \mu^{+}\right\|_{\infty}<\infty
$$

and so $(X, \mu)$ is gaugeable by Corollary [2.9, This proves that (1) and (2) are equivalent.

To see that (2) and (3) are equivalent, note that by Lemma 3.5(1),

$$
V 1_{B}\left(x_{0}\right)=\int_{B} G\left(x_{0}, y\right) \mathbf{E}_{x_{0}}^{y}\left[e_{A}\left(\zeta^{y}\right)\right] m(d y) .
$$

If $V 1_{B}\left(x_{0}\right)<\infty$ for some $x_{0} \in E$ and some Borel set $B$ with $m(B)>0$, then $\mathbf{E}_{x_{0}}^{y}\left[e_{A}\left(\zeta^{y}\right)\right]<\infty$ for some $y \in E$ satisfying $0<G\left(x_{0}, y\right)<\infty$. Therefore by Theorem 3.3, $(X, \mu)$ is conditionally gaugeable. Conversely, if $(X, \mu)$ is conditionally gaugeable, then $\mathbf{E}_{x}^{y}\left[e_{A}\left(\zeta^{y}\right)\right]$ is bounded on $(E \times E) \backslash d$. Since $X$ is transient, by Getoor [23, there is a set $B \subset E$ of positive $m$-measure such that $G 1_{B}$ is bounded and therefore $V 1_{B}$ is bounded. This finishes the proof of the equivalence between (2) and (3).

Note that under the condition of Theorem 3.6, if $\mathcal{L}+\mu$ is subcritical, then by Jensen's inequality, Lemma [3.5] and Theorem [3.6, the Green function of $\mathcal{L}+\mu$ is comparable to that of $\mathcal{L}$. 
The above definition of subcriticality for the Schrödinger operator $\mathcal{L}+\mu$ is formulated under the circumstance that we do not know if the resolvent density function $V(x, y)$ can be uniquely defined pointwise. Suppose now that

$$
\begin{aligned}
& X_{t} \text { has continuous transition density function } p(t, x, y) \\
& \text { with } \sup _{x, y \in E} p(t, x, y)<\infty,
\end{aligned}
$$

and that $\mu$ is a signed measure in $\mathbf{S}_{\infty}(X)$. Then by the same argument as that for Theorem 3.17 in Chung and Zhao [13], $Q_{t}$ has a continuous density function $q(t, x, y)$ which is strictly positive on $\mathbf{R}_{+} \times E \times E$. In this case $V(x, y):=\int_{0}^{\infty} q(t, x, y) d t$ is pointwise defined and is the density kernel for the resolvent $V$. Clearly if $V 1_{B}\left(x_{0}\right)<$ $\infty$ for some $x_{0} \in E$ and some subset $B \subset E$ with $m(B)>0$, then $V\left(x_{0}, y_{0}\right)<\infty$ for some $\left(x_{0}, y_{0}\right) \in(E \times E) \backslash d$. Conversely, if $V\left(x_{0}, y_{0}\right)<\infty$ for some $\left(x_{0}, y_{0}\right) \in(E \times$ $E) \backslash d$, then $\int_{E} V\left(x_{0}, z\right) q\left(1, z, y_{0}\right) m(d z) \leq V\left(x_{0}, y_{0}\right)<\infty$. Since $z \mapsto q\left(1, z, y_{0}\right)>0$ is continuous, there is $r>0$ such that $V 1_{B\left(y_{0}, r\right)}\left(x_{0}\right)<\infty$. So we have the following.

Proposition 3.7. Assume that condition (3.13) holds and that $\mu$ is a signed smooth measure in $\mathbf{S}_{\infty}(X)$. Then $\mathcal{L}+\mu$ is subcritical if and only if $V\left(x_{0}, y_{0}\right)<\infty$ for some $\left(x_{0}, y_{0}\right) \in(E \times E) \backslash d$.

Remark 3.1. If the Schrödinger semigroup $Q_{t}$ defined by (3.9) has positive $L^{2}-$ spectral radius, that is, if

$$
\lambda_{2}(X, \mu):=-\lim _{t \rightarrow \infty} \frac{1}{t} \log \left\|Q_{t}\right\|_{2,2}>0,
$$

then its resolvent $V=\int_{0}^{\infty} Q_{t} d t$ is a bounded operator in $L^{2}(E, m)$. This implies that $\mathcal{L}+\mu$ is subcritical by applying $V$ to the function $f=1_{B}$ for some Borel subset with $0<m(B)<\infty$. Hence under the condition of Theorem [3.6. $(X, \mu)$ is gaugeable. We see from Theorem 2.12 that when $X$ is symmetric and the state space $E$ satisfies certain conditions, $\lambda_{2}(X, \mu)>0$ is a necessary and sufficient condition for $(X, \mu)$ to be gaugeable. But in general $\lambda_{2}(X, \mu)>0$ is not a necessary condition for $(X, \mu)$ being gaugeable, even when $X$ is a Brownian motion in $\mathbf{R}^{n}$. See Remark 5.1 below.

3.2. Conditional Gauge Theorem for Non-local Perturbations. We now turn to the conditional gauge theorem for discontinuous additive functionals. In this subsection, we assume further that $X$ is a Borel standard process having a strong dual Borel standard process $\left(\widehat{X}, \widehat{\mathbf{P}}_{x}, x \in E\right)$ on $E$ with respect to the measure $m$. For any excessive function $h$ of $X$, it is known from Kunita and Watanabe [27] and Proposition 2.2 of Fitzsimmons [18] that Doob's $h$-transformed process $X^{h}$ is Borel standard on $E_{h}$. Recall that $(N, H)$ is the Lévy system of $X$ and $\mu_{H}$ is the Revuz measure for the PCAF $H$ of $X$.

Definition 3.4. Suppose $F$ is a bounded function on $E \times E$ vanishing on the diagonal. Define $\mu_{|F|}(d x):=\left(\int_{E}|F(x, y)| N(x, d y)\right) \mu_{H}(d x)$. Function $F$ is said to be in the class $\mathbf{A}_{\infty}(X)$ if for any $\varepsilon>0$ there is a Borel subset $K=K(\varepsilon)$ of finite $\mu_{|F|}$-measure and a constant $\delta=\delta(\varepsilon)>0$ such that

$$
\sup _{(x, w) \in(E \times E) \backslash d} \int_{(K \times K)^{c}} G(x, y) \frac{|F(y, z)| G(z, w)}{G(x, w)} N(y, d z) \mu_{H}(d y) \leq \varepsilon
$$


and for all measurable sets $B \subset K$ with $\mu_{|F|}(B)<\delta$,

$$
\sup _{(x, w) \in(E \times E) \backslash d} \int_{(B \times E) \cup(E \times B)} G(x, y) \frac{|F(y, z)| G(z, w)}{G(x, w)} N(y, d z) \mu_{H}(d y) \leq \varepsilon .
$$

The corresponding Kato class introduced by Chen and Song in [9], which will now be denoted as $\widetilde{\mathbf{A}}_{\infty}(X)$, is defined very similar to that of $\mathbf{A}_{\infty}(X)$ above except conditions involving measure $\mu_{|F|}$ are replaced by the measure $m$. As seen from the last section, the new $\mathbf{A}_{\infty}(X)$ is an extension of $\widetilde{\mathbf{A}}_{\infty}(X)$, which allows us to cover the cases where $\mu_{H}$ may not be absolutely continuous with respect to $m$. By a similar proof as that for Corollary 3.2 in [9], we have $\mathbf{A}_{\infty}(X) \subset \mathbf{J}_{\infty}(X)$. Note that by Proposition 3.2 and the definition of $\mathbf{A}_{\infty}(X)$, for $\mu \in \mathbf{S}_{1}(X)$ and $F \in \mathbf{A}_{\infty}(X)$,

$$
\sup _{(x, w) \in(E \times E) \backslash d} \mathbf{E}_{x}^{w}\left[A_{\zeta^{w}}^{|\mu|}+\sum_{0<s<\zeta^{w}}\left|F\left(X_{s-}^{w}, X_{s}^{w}\right)\right|\right]<\infty .
$$

Thus $e_{A^{\mu}+F}\left(\zeta^{w}\right)$ is well defined under $\mathbf{P}_{x}^{w}$, where $e_{A^{\mu}+F}(t)$ is given by (2.23). Furthermore, by Jensen's inequality,

$$
\inf _{(x, w) \in(E \times E) \backslash d} \mathbf{E}_{x}^{w}\left[e_{A^{\mu}+F}\left(\zeta^{w}\right)\right]>0 .
$$

Theorem 3.8 (Conditional Gauge Theorem II). Suppose that $\mu \in \mathbf{S}_{1}(X)$ and $F \in$ $\mathbf{A}_{\infty}(X)$. If $\mathbf{E}_{x}^{w}\left[e_{A^{\mu}+F}\left(\zeta^{w}\right)\right]$ is finite for some $\left(x_{0}, w_{0}\right) \in(E \times E) \backslash d$, then $\mathbf{E}_{x}^{w}\left[e_{A^{\mu}+F}\left(\zeta^{w}\right)\right]$ is bounded on $(E \times E) \backslash d$. Here $e_{A^{\mu}+F}(t)$ is given by (2.23).

Proof. The proof is similar to that for Theorem 3.5 in Chen and Song [9] with suitable modifications. So it is omitted here.

Definition 3.5. For a signed smooth measure $\mu \in \mathbf{S}_{1}(X)$ and $F \in \mathbf{A}_{\infty}(X)$, we say $\left(X, A^{\mu}+F\right)$ is conditionally gaugeable if the conditional gauge function $(x, w) \mapsto$ $\mathbf{E}_{x}^{w}\left[e_{A^{\mu}+F}\left(\zeta^{w}\right)\right]$ is bounded on $(E \times E) \backslash d$.

For $\mu \in \mathbf{K}_{1}(X)$ and $F \in \mathbf{J}_{\infty}(X)$, let $A$ be the continuous additive functional of $X$ with Revuz measure $\mu$ and define the Schrödinger semigroup

$$
Q_{t} f(x):=\mathbf{E}_{x}\left[e_{A+F}(t) f\left(X_{t}\right)\right] \text { for } f \geq 0 \text {. }
$$

Its resolvent operator $V$ is defined as

$$
V f(x):=\int_{0}^{\infty} Q_{t} f(x)=\mathbf{E}_{x}\left[\int_{0}^{\infty} e_{A+F}(t) f\left(X_{t}\right) d t\right] .
$$

As was mentioned earlier in section 2 , the infinitesimal generator for the semigroup $Q_{t}$ is formally $\mathcal{L}+\mu_{H} \mathbf{F}+\mu$, where the non-local operator $\mathbf{F}$ is defined by (2.26).

Analogous to Lemma [3.5] and with almost the identical proof, we have the following.

Lemma 3.9. Suppose that $\mu \in \mathbf{K}_{1}(X)$ and $F \in \mathbf{J}_{\infty}(X)$.

(1) For every Borel function $f \geq 0$,

$$
\mathbf{E}_{x}\left[\int_{0}^{\infty} e_{A^{\mu}+F}(t) f\left(X_{t}\right) d t\right]=\int_{E} G(x, y) \mathbf{E}_{x}^{y}\left[e_{A^{\mu}+F}\left(\zeta^{y}\right)\right] f(y) m(d y) .
$$

That is, $V(x, y):=G(x, y) \mathbf{E}_{x}^{y}\left[e_{A^{\mu}+F}\left(\zeta^{y}\right)\right]$ is the Green function of $\mathcal{L}+\mu_{H} \mathbf{F}+\mu$. 
(2) For any positive measure $\nu \in \mathbf{K}_{1}(X)$,

$$
\mathbf{E}_{x}\left[\int_{0}^{\infty} e_{A^{\mu}+F}(t) d A_{t}^{\nu}\right]=\int_{E} G(x, y) \mathbf{E}_{x}^{y}\left[e_{A^{\mu}+F}\left(\zeta^{y}\right)\right] \nu(d y),
$$

where $A^{\nu}$ is the PCAF of $X$ with Revuz measure $\nu$.

Definition 3.6. We call the Schrödinger operator $\mathcal{L}+\mu_{H} \mathbf{F}+\mu$ subcritical if there is some $x_{0} \in E$ and a Borel subset $B \subset E$ of positive $m$-measure such that $V 1_{B}\left(x_{0}\right)<$ $\infty$, where the resolvent $V$ is defined by (3.17).

Theorem 3.6 has the following extension for the non-local perturbation $\left(X, A^{\mu}+\right.$ $F)$.

Theorem 3.10. Suppose that $\mu \in \mathbf{S}_{1}(X)$ and $F \in \mathbf{A}_{\infty}(X)$. Then the following are equivalent:

(1) $\left(X, A^{\mu}+F\right)$ is gaugeable;

(2) $\left(X, A^{\mu}+F\right)$ is conditionally gaugeable;

(3) $\mathcal{L}+\mu_{H} \mathbf{F}+\mu$ is subcritical.

Proof. Note that $\mathbf{S}_{1}(X) \subset \mathbf{K}_{1}(X)$ and $\mathbf{A}_{\infty}(X) \subset \mathbf{J}_{\infty}(X)$. Let $A^{+}$and $A^{-}$be the PCAFs of $X$ with Revuz measures $\mu^{+}$and $\mu^{-}$respectively. Let $A=A^{+}-A^{-}$, which is $A^{\mu}$.

Suppose $(X, A+F)$ is gaugeable. Then by Lemma $3.9(2)$,

$$
\begin{aligned}
\int_{E} G(x, y) \mathbf{E}_{x}^{y}\left[e_{A+F}\left(\zeta^{y}\right)\right]\left(\mu^{+}(d y)+N F^{+}(y) \mu_{H}(d y)\right) \\
\left.\quad=\mathbf{E}_{x}\left[\int_{0}^{\infty} e_{A+F}(t) d A_{t}^{+}+\int_{0}^{\infty} e_{A+F}(t) N F^{+}\left(X_{t}\right) d H_{t}\right)\right] .
\end{aligned}
$$

Since $F$ is bounded and $\int_{0}^{t} N F^{+}\left(X_{s}\right) d H_{s}$ is the dual predictable projection of $A_{t}^{F^{+}}$, we have

Thus by Corollary 2.16

$$
\begin{aligned}
\mathbf{E}_{x}\left[\int_{0}^{\infty} e_{A+F}(t) N F^{+}\left(X_{t}\right) d H_{t}\right] & \approx \mathbf{E}_{x}\left[\int_{0}^{\infty} e_{A+F}(t-) N F^{+}\left(X_{t}\right) d H_{t}\right] \\
& =\mathbf{E}_{x}\left[\int_{0}^{\infty} e_{A+F}(t-) d A_{t}^{F^{+}}\right] .
\end{aligned}
$$

$$
\begin{gathered}
\int_{E} G(x, y) \mathbf{E}_{x}^{y}\left[e_{A+F}\left(\zeta^{y}\right)\right]\left(\mu^{+}(d y)+N F^{+}(y) \mu_{H}(d y)\right) \\
\approx \mathbf{E}_{x}\left[\int_{0}^{\infty} e_{A+F}(t-) d\left(A_{t}^{+}+A_{t}^{F^{+}}\right)\right]<\infty .
\end{gathered}
$$

Therefore there is some $(x, y) \in(E \times E) \backslash d$ such that $\mathbf{E}_{x}^{y}\left[e_{A+F}\left(\zeta^{y}\right)\right]<\infty$ and so by Theorem $3.8(X, A+F)$ is conditionally gaugeable. Conversely, if $(X, A+F)$ is conditionally gaugeable, it follows from the above calculation that

$$
\sup _{x \in E}\left[\int_{0}^{\infty} e_{A+F}(t) d\left(A_{t}^{+}+A_{t}^{F^{+}}\right)\right] \leq c\left\|G\left(\mu^{+}+\left(N F^{+}\right) \mu_{H}\right)\right\|_{\infty}<\infty
$$

and so $(X, A+F)$ is gaugeable by Corollary 2.16. This proves that (1) and (2) are equivalent.

To see that (2) and (3) are equivalent, note that by Lemma 3.5(1),

$$
V 1_{B}\left(x_{0}\right)=\int_{B} G\left(x_{0}, y_{0}\right) \mathbf{E}_{x_{0}}^{y}\left[e_{A+F}\left(\zeta^{y}\right)\right] m(d y) .
$$


If $V 1_{B}\left(x_{0}\right)<\infty$ for some $x_{0} \in E$ and some Borel set $B$ with $m(B)>0$, then $\mathbf{E}_{x_{0}}^{y}\left[e_{A+F}\left(\zeta^{y}\right)\right]<\infty$ for some $y \in E$ satisfying $0<G\left(x_{0}, y\right)<\infty$. Therefore by Theorem [3.8. $(X, A+F)$ is conditionally gaugeable. Conversely, if $(X, A+F)$ is conditionally gaugeable, then $\mathbf{E}_{x}^{y}\left[e_{A+F}\left(\zeta^{y}\right)\right]$ is bounded on $(E \times E) \backslash d$. Since $X$ is transient, by Getoor [23], there is a set $B \subset E$ of positive $m$-measure such that $G 1_{B}$ is bounded and therefore $V 1_{B}$ is bounded. This finishes the proof of the equivalence between (2) and (3).

Under the condition of Theorem 3.10, if $\mathcal{L}+\mu_{H} \mathbf{F}+\mu$ is subcritical, then by (3.16), Lemma 3.9 and Theorem 3.10, the Green function of $\mathcal{L}+\mu_{H} \mathbf{F}+\mu$ is comparable to that of $\mathcal{L}$.

Noting that if $\mu \in \mathbf{S}_{1}(X)$, there is an $\varepsilon>0$ such that $(1+\varepsilon)|\mu| \in \mathbf{S}_{1}(X)$, the next result follows immediately from Theorem 2.17 and Theorem 3.10 .

Theorem 3.11 (Super Conditional Gauge Theorem). Suppose that $\mu \in \mathbf{S}_{1}(X)$, $F \in \mathbf{A}_{\infty}(X)$ and that $\left(X, A^{\mu}+F\right)$ is conditionally gaugeable. Then there is an $\varepsilon_{0}>0$ such that $\left(X, A^{\mu+\varepsilon_{0}|\mu|}+F+\varepsilon_{0}|F|\right)$ is conditionally gaugeable. In particular, $\left(X, A^{(1+\varepsilon) \mu}+(1+\varepsilon) F\right)$ is conditionally gaugeable for all $\varepsilon \in\left[0, \varepsilon_{0}\right]$.

Remark 3.2. Theorem [3.4 can also be extended to $\left(X, A^{\mu}+F\right)$ where $\mu \in \mathbf{S}_{1}(X)$ and $F \in \mathbf{J}_{\infty}(X)$, with $A+F$ and $A^{+}+A^{F^{+}}$in place of $A$ and $A^{+}$respectively. The proof is along the same line of reasoning as that for Theorem 3.4, so we omit the details here.

\section{4. $L^{p}$-INDEPENDENCE OF SPECTRAL RADIUS}

In this section, $X$ is an irreducible transient Borel right process with strong dual $\widehat{X}$ and has Green function $G(x, y)$ with respect to $m$, as given at the beginning of the last section. For $p \geq 1$, define the $L^{p}$-spectral radius

$$
\lambda_{p}:=-\lim _{t \rightarrow \infty} \frac{1}{t} \log \left\|P_{t}\right\|_{p, p} .
$$

Here $\left\|P_{t}\right\|_{p, p}$ is the operator norm of $P_{t}: L^{p}(E, m) \rightarrow L^{p}(E, m)$. Clearly

$$
\left\|P_{t}\right\|_{\infty, \infty}=\sup _{x \in E} \mathbf{P}_{x}(t<\zeta) .
$$

Since

$$
\left|P_{t} f(x)\right| \leq \sqrt{P_{t}\left(f^{2}\right)(x)} \sqrt{\mathbf{P}_{x}(t<\zeta)}
$$

we have

$$
\left\|P_{t} f\right\|_{2}^{2} \leq\left\|P_{t}\left(f^{2}\right)\right\|_{1} \sup _{x \in E} \mathbf{P}_{x}(t<\zeta) \leq \sup _{x \in E} \mathbf{P}_{x}(t<\zeta)\|f\|_{2}^{2} .
$$

The last inequality is due to the fact that $\left\|P_{t}\left(f^{2}\right)\right\|_{1}=\int_{E} f(x)^{2} \widehat{P}_{t} 1(x) m(d x) \leq$ $\|f\|_{2}^{2}$. Hence

$$
\left\|P_{t}\right\|_{2,2} \leq \sup _{x \in E} \mathbf{P}_{x}(t<\zeta)=\left\|P_{t}\right\|_{\infty, \infty}
$$

Using the Riesz interpolation theorem, we have

$$
\left\|P_{t}\right\|_{2,2} \leq\left\|P_{t}\right\|_{p, p} \leq\left\|P_{t}\right\|_{\infty, \infty} \quad \text { for } 2 \leq p<\infty .
$$

This implies that

$$
\lambda_{2} \geq \lambda_{p} \geq \lambda_{\infty} \quad \text { for all } p \geq 2 .
$$


If $X$ is a symmetric process, then by symmetry of $P_{t}$, the last two inequalities hold for all $p \geq 1$.

Applying Theorem 3.1, Corollary 3.2 and Lemma 3.5 of Sato 32 to the 1subprocess of $X$, we conclude

$$
\sup _{x \in E} \mathbf{E}_{x}\left[e^{\lambda \zeta}\right]<\infty \quad \text { if and only if } \lambda<\lambda_{\infty} .
$$

Theorem 4.1 (Independence of $L^{p}$-Spectral Radius). Suppose that the constant function $1 \in \mathbf{K}_{\infty}(X)$. Then $\lambda_{2}=\lambda_{\infty}$. That is, the spectral radius $\lambda_{p}$ is independent of $p \in[2, \infty]$.

Proof. Note that $\lambda_{\infty} \leq \lambda_{2}$ and hence we only need to show that if $\lambda<\lambda_{2}$, then $\lambda<\lambda_{\infty}$. For this, without loss of generality, we may assume $0<\lambda<\lambda_{2}$. Let $\varepsilon>0$ and $q>1$ be such that $\lambda+\varepsilon<\lambda_{2}$ and $\frac{\lambda}{\lambda+\varepsilon}+\frac{1}{q}=1$. It follows from the definition of $\lambda_{2}$ that $\left\|G_{-(\lambda+\varepsilon)}\right\|_{2,2}<\infty$, where $G_{-a}=\int_{0}^{\infty} e^{a s} P_{s} d s$. Since $1 \in \mathbf{K}_{\infty}(X)$, by Definition 2.2, there is an open set $K$ of finite $m$-measure such that $\sup _{x \in E} G 1_{K^{c}}(x) \leq(2 \lambda q)^{-1}$. Since $1_{K} \in L^{2}(E, m)$, the function

$$
G_{-(\lambda+\varepsilon)} 1_{K}(x)=\mathbf{E}_{x}\left[\int_{0}^{\infty} e^{(\lambda+\varepsilon) s} 1_{K}\left(X_{s}\right) d s\right]
$$

is $L^{2}$-integrable. Using the elementary inequality

$$
e^{(\lambda+\varepsilon) a}-e^{(\lambda+\varepsilon) b} \geq e^{(\lambda+\varepsilon)(a-c)}-e^{(\lambda+\varepsilon)(b-c)} \quad \text { for } a>b>c \geq 0,
$$

we have

$$
1+(\lambda+\varepsilon) G_{-(\lambda+\varepsilon)} 1_{K}(x) \geq \mathbf{E}_{x}\left[\exp \left((\lambda+\varepsilon) \int_{0}^{\infty} 1_{K}\left(X_{s}\right) d s\right)\right] .
$$

Now by Hölder's inequality,

$$
\begin{aligned}
\mathbf{E}_{x}\left[e^{\lambda \zeta}\right]= & \mathbf{E}_{x}\left[\exp \left(\lambda \int_{0}^{\infty} 1_{K^{c}}\left(X_{s}\right) d s\right) \exp \left(\lambda \int_{0}^{\infty} 1_{K}\left(X_{s}\right) d s\right)\right] \\
\leq & \left(\mathbf{E}_{x}\left[\exp \left(q \lambda \int_{0}^{\infty} 1_{K^{c}}\left(X_{s}\right) d s\right)\right]\right)^{1 / q} \\
& \quad \times\left(\mathbf{E}_{x}\left[\exp \left((\lambda+\varepsilon) \int_{0}^{\infty} 1_{K}\left(X_{s}\right) d s\right)\right]\right)^{\lambda /(\lambda+\varepsilon)} \\
\leq & 2^{1 / q}\left(1+(\lambda+\varepsilon) G_{-(\lambda+\varepsilon)} 1_{K}(x)\right)^{\lambda /(\lambda+\varepsilon)} .
\end{aligned}
$$

In the last inequality, we used Khasminskii's inequality. Thus $\mathbf{E}_{x}\left[e^{\lambda \zeta}\right]<\infty m$-a.e. on $E$ and therefore by Theorem 2.6, $\sup _{x \in E} \mathbf{E}_{x}\left[e^{\lambda \zeta}\right]<\infty$. This implies $\lambda<\lambda_{\infty}$ and so $\lambda_{2}=\lambda_{\infty}$.

Theorem 4.2. Suppose that $X$ has the strong Feller property (that is, $G_{1}$ maps bounded Borel measurable functions into bounded continuous functions) and that for every $\varepsilon>0$, there is a compact set $K$ such that $\sup _{x \in E} G_{1} 1_{K^{c}}(x) \leq \varepsilon$. Then $1 \in \mathbf{K}_{\infty}(Y)$, where $Y$ is the 1-subprocess of $X$. In particular, this implies that $\lambda_{2}=\lambda_{\infty}$.

Proof. First note that the strong Feller property implies that the resolvent kernel $G_{1}(x, d y)$ is absolutely continuous with respect to $m$. Let $G^{Y}$ be the Green function for $Y$. Then clearly $G^{Y}=G_{1}$. For any $\varepsilon>0$, let $K$ be the compact set such that $\sup _{x \in E} G_{1} 1_{K^{c}}(x) \leq \varepsilon$. We claim that there is a constant $\delta>0$ such that for any 
Borel measurable subset $B \subset K$ with $m(B)<\delta$, $\sup _{x \in E} G_{1} 1_{B}(x) \leq \varepsilon$. Suppose that this is not true. Then there is a decreasing sequence of Borel measurable subsets $B_{k}$ of $K$ with $m\left(B_{k}\right)<1 / k$ such that $\sup _{x \in E} G_{1} 1_{B_{k}}(x) \geq \varepsilon$ for each $k \geq 1$. By the strong Markov property,

$$
\sup _{x \in E} G_{1} 1_{B_{k}}(x)=\sup _{x \in K} G_{1} 1_{B_{k}}(x) .
$$

Since $G_{1} 1_{B_{k}}$ is a bounded continuous function and $K$ is compact, there is $x_{k} \in K$ so that

$$
G_{1} 1_{B_{k}}\left(x_{k}\right)=\sup _{x \in E} G_{1} 1_{B_{k}}(x) \geq \varepsilon .
$$

Taking a subsequence if necessary, we may assume that $x_{k} \rightarrow x_{0} \in K$. Since $G_{1} 1_{B_{k}}\left(x_{0}\right)$ decreases to 0 as $k \uparrow 0$, there is $k_{0}$ so that $G_{1} 1_{B_{k_{0}}}\left(x_{0}\right)<\varepsilon / 3$. By the continuity of $x \mapsto G_{1} 1_{B_{k_{0}}}(x)$, there is a neighborhood $U$ of $x_{0}$ such that $\sup _{x \in U} G_{1} 1_{B_{k_{0}}}(x)<\varepsilon / 2$. As $x_{k} \rightarrow x_{0}, x_{k} \in U$ when $k>k_{0}$ is sufficiently large and so

$$
G_{1} 1_{B_{k}}\left(x_{k}\right) \leq G_{1} 1_{B_{k_{0}}}\left(x_{k}\right)<\varepsilon / 2
$$

which contradicts (4.3). This proves the claim and therefore $1 \in \mathbf{K}_{\infty}(Y)$. Now by Theorem 4.1, the spectral radius $\lambda_{p}(Y)$ of $Y$ is independent of $p \in[2, \infty]$. Since $Y$ is the 1 -subprocess of $X, \lambda_{p}(Y)=\lambda_{p}+1$. Thus the spectral radius $\lambda_{p}$ of $X$ is independent of $p \in[2, \infty]$.

Recently Takeda [40] proved the $L^{p}$-independence of the spectral radius $\lambda_{p}$ under the conditions of Theorem 4.2 with an additional assumption that $X$ is $m$ symmetric, using a Donsker-Varadhan type large deviation principle approach.

\section{Analytic Characterizations FOR GaUgeability AND CONDITIONAL GAUGEABILITY}

In addition to the assumption made at tbe beginning of section 3 , we assume in this section that the process $X$ is symmetric with respect to the measure $m$ and therefore, by results due to Albeverio-Ma and Fitzsimmons (see [28]), its associated Dirichlet form $(\mathcal{E}, \mathcal{F})$ is quasi-regular on $L^{2}(E, m)$. It is shown in Chen, Ma and Röckner [5] that such a process $X$ is quasi-homeomorphic to an $m$-symmetric irreducible transient Hunt process on a locally compact separable metric space whose Dirichlet form is regular. So without loss of generality, we assume that $X$ is an $m$ symmetric irreducible transient Hunt process on a locally compact separable metric space $E$ whose Dirichlet $(\mathcal{E}, \mathcal{F})$ form is regular. It is well known that its $L^{2}$-spectral radius $\lambda_{2}$ has the following analytic characterization:

$$
\lambda_{2}=\inf \left\{\mathcal{E}(u, u): u \in \mathcal{F} \text { with } \int_{E} u(x)^{2} m(d x)=1\right\} .
$$

We will use the result of $L^{p}$-independence of the spectral radius $\lambda_{p}$ from the last section to give analytic characterizations of gaugeability and conditional gaugeability in terms of the associated bilinear forms, extending the results in Takeda [40].

For a signed smooth measure $\mu$, let $A^{\mu}$ be the continuous additive functional of $X$ with Revuz measure $\mu$. Define $e_{A}(t):=\exp \left(A_{t}^{\mu}\right)$ and the gauge function $g_{\mu}(x):=\mathbf{E}_{x}\left[e_{A}(\zeta)\right]$. Recall that by Theorem [2.6] if $\mu$ is in $\mathbf{K}_{1}(X)$, then $g_{\mu}$ is either identically infinite or bounded on $E$. 
The following theorem was proved in Takeda [40] under some additional continuity and asymptotic assumptions (Assumption $\mathrm{G}$ there) on the Green function $G(x, y)$. The proof of our result is almost the same as that for Theorem 2.4 in [40]. For the reader's convenience, we spell out the details here.

Theorem 5.1. Let $\mu$ be a positive measure in $\mathbf{K}_{\infty}(X)$. Then $(X, \mu)$ is gaugeable if and only if

$$
\inf \left\{\mathcal{E}(u, u): u \in \mathcal{F} \text { with } \int_{E} u(x)^{2} \mu(d x)=1\right\}>1 .
$$

Proof. Let $\tau_{t}$ be the right continuous inverse of $A_{t}^{\mu}$; that is,

$$
\tau_{t}=\inf \left\{s: A_{s}^{\mu}>t\right\}
$$

with the convention that $\inf \emptyset=\infty$. Let $\widetilde{S}:=\left\{x \in E: \mathbf{P}_{x}\left(\tau_{0}=0\right)=1\right\}$ be the fine support of $\mu$ and let $S$ be the topological support of $\mu$. The time-changed process $Y_{t}^{\mu}$ of $X_{t}$ by $A^{\mu}$ is defined by $Y_{t}^{\mu}=X_{\tau_{t}}$, whose state space is $\widetilde{S}$. However, since $\widetilde{S} \subset S$ modulo a set having zero capacity, the semigroup of $Y^{\mu}$ is $\mu$-symmetric and determines a strongly continuous semigroup on $L^{2}(Y, \mu)$ (cf. Theorem 6.2.1 of 22]). So this time-changed process $Y^{\mu}$ is a $\mu$-symmetric right process. Set $H_{S} u(x):=\mathbf{E}_{x}\left[u\left(X_{\sigma_{S}}\right)\right]$, where $\sigma_{S}=\inf \left\{t>0: X_{t} \in S\right\}$. Then the Dirichlet form $(\widehat{\mathcal{E}}, \widehat{\mathcal{F}})$ of $Y^{\mu}$ on $L^{2}(S, \mu)$ is given by

$$
\left\{\begin{array}{r}
\widehat{\mathcal{F}}=\left\{\varphi \in L^{2}(S ; \mu): \varphi=u \mu \text {-a.e. on } S \text { for some } u \in \mathcal{F}_{e}\right\} \\
\widehat{\mathcal{E}}(\varphi, \varphi)=\mathcal{E}\left(H_{S} u, H_{S} u\right), \varphi \in \widehat{\mathcal{F}} \text { and } u \in \mathcal{F}_{e} \\
\text { such that } \varphi=u \mu \text {-a.e. on } S .
\end{array}\right.
$$

Here $\mathcal{F}_{e}$ stands for the extended Dirichlet space of $(\mathcal{E}, \mathcal{F})$. Note that for every Borel $f \geq 0$,

$$
\begin{aligned}
\mathbf{E}_{x}\left[\int_{0}^{\infty} f\left(Y_{t}^{\mu}\right) d t\right] & =\mathbf{E}_{x}\left[\int_{0}^{\infty} f\left(X_{\tau_{t}}\right) d t\right]=\mathbf{E}_{x}\left[\int_{0}^{\infty} f\left(X_{t}\right) d A_{t}^{\mu}\right] \\
& =\int_{S} G(x, y) f(y) \mu(d y) .
\end{aligned}
$$

So the Green function of $Y^{\mu}$ with respect to $\mu$ is $G(x, y)$. Hence the constant function $1 \in \mathbf{K}_{\infty}\left(Y^{\mu}\right)$. Since $A_{\zeta}^{\mu}$ is the lifetime of the time-changed process $Y$, by Theorem 4.1.

$$
\sup _{x \in S} \mathbf{E}_{x}\left[e^{A_{\zeta}^{\mu}}\right]<\infty \text { if and only if } \lambda_{2}(Y)>1
$$

Note that

$$
\lambda_{2}(Y)=\inf \left\{\widehat{\mathcal{E}}(\varphi, \varphi): \varphi \in \widehat{\mathcal{F}} \text { with } \int_{S} \varphi(x)^{2} \mu(d x)=1\right\},
$$

which by Lemma 3.1 of Takeda 39 is the same as

$$
=\inf \left\{\mathcal{E}(u, u): u \in \mathcal{F} \text { with } \int_{E} u(x)^{2} \mu(d x)=1\right\} .
$$

The theorem is now proved.

Using Theorem [2.10] we can extend the above theorem to the case where the potential $\mu$ is a signed measure. 
Theorem 5.2. Suppose that $\mu$ is a signed smooth measure such that $\mu^{+} \in \mathbf{K}_{\infty}(X)$ and $G \mu^{-}$is bounded. Then $(X, \mu)$ is gaugeable if and only if

$$
\inf \left\{\mathcal{E}(u, u)+\int_{E} u(x)^{2} \mu^{-}(d x): u \in \mathcal{F} \text { with } \int_{E} u(x)^{2} \mu^{+}(d x)=1\right\}>1 .
$$

Proof. Let $A^{+}$and $A^{-}$be the PCAFs of $X$ with Revuz measures $\mu^{+}$and $\mu^{-}$ respectively. Let $Y$ be the subprocess of $X$ killed at rate $\mu^{-}$; in other words, $Y$ is obtained from $X$ through the Feynman-Kac transform $e^{-A_{t}^{-}}$. It is known (cf. Fitzsimmons [18]) that $Y$ is a symmetric Markov process on $L^{2}(E, m)$ with Dirichlet form $\left(\mathcal{E}^{Y}, \mathcal{F}\right)$, where

$$
\mathcal{E}^{Y}(u, v)=\mathcal{E}(u, v)+\int_{E} u(x) v(x) \mu^{-}(d x) \quad \text { for } u, v \in \mathcal{F} .
$$

By Fitzsimmons and Getoor [19], $X$ and $Y$ have the same class of smooth measures; in particular, $A^{+}$can be viewed as the continuous additive functional of $Y$ with Revuz measure $\mu^{+}$. It is clear that the Green function $G^{Y}(x, y)$ of $Y$ satisfies $G^{Y}(x, y) \leq G(x, y)$. Therefore $\mu^{+} \in \mathbf{K}_{\infty}(X) \subset \mathbf{K}_{\infty}(Y)$ and $G^{Y} \mu^{-}$is bounded. By Theorem 2.10, $(X, \mu)$ is gaugeable if and only if $\left(Y, \mu^{+}\right)$is gaugeable. The latter is equivalent to (5.3) by Theorem 5.1 and (5.4).

Putting Theorem 3.6 and Theorem 5.2 together yields the following.

Theorem 5.3. Suppose that $\mu$ is a signed smooth measure such that $\mu^{+} \in \mathbf{S}_{\infty}(X)$ and

$$
\sup _{(x, w) \in(E \times E) \backslash d} \int_{E_{w}} \frac{G(x, y) G(y, w)}{G(x, w)} \mu^{-}(d y)<\infty .
$$

Then the following are equivalent:

(1) $(X, \mu)$ is gaugeable;

(2) $(X, \mu)$ is conditionally gaugeable;

(3) Schrödinger operator $\mathcal{L}+\mu$ is subcritical;

(4) Inequality (5.3) holds.

Remark 5.1. The $L^{2}$-spectral radius characterization of the gaugeability of $(X, \mu)$ given in Theorem 2.12 while very useful, requires the state space $E$ to have finite $m$-mass and be Green-bounded. The characterization given in Theorem 5.2 does not have this restriction. Note that Theorem 2.12 fails if its condition on the state space $E$ is not satisfied. For example, let $E=\mathbf{R}^{n}$ with $n \geq 3$, let $X$ be a Brownian motion on $\mathbf{R}^{n}$ and let $\mu$ be the surface measure of a sphere with radius $R$. Then clearly $\lambda_{2}(X, \mu) \leq 0$ since the bottom of the spectrum for the Laplacian in $\mathbf{R}^{n}$ is zero. However, using the analytic characterization (5.1), it was shown in Takeda [40] that $(X, \mu)$ is gaugeable if and only if the radius $R<(n-2) / 2$.

However, under the condition of Theorem 5.3. as we noted in Remark 5.1, if $\lambda_{2}(X, \mu)>0$, then $(X, \mu)$ is gaugeable and hence inequality (5.3) holds. This fact, that condition $\lambda_{2}(X, \mu)>0$ implies that inequality (5.3) holds, has been observed in 40 using a bilinear form technique.

Using Theorems 5.2 and 5.3 we can give an alternative proof for Theorems 2.17 and 3.11 when the Feynman-Kac transforms are given by continuous additive functionals. 
Theorem 5.4. (1) (Super Gauge Theorem) Under the conditions of Theorem 5.2, if $(X, \mu)$ is gaugeable, then there is an $\varepsilon_{0}>0$ such that $\left(X, \mu+\varepsilon_{0}|\mu|\right)$ is gaugeable. In particular, $(X,(1+\varepsilon) \mu)$ is gaugeable for all $\varepsilon \in\left[0, \varepsilon_{0}\right]$.

(2) (Super Conditional Gauge Theorem) Under the conditions of Theorem 5.3, if $(X, \mu)$ is conditionally gaugeable, then there is an $\varepsilon_{0} \in(0,1)$ such that $\left(X, \mu+\varepsilon_{0}|\mu|\right)$ is conditionally gaugeable. In particular, $(X,(1+\varepsilon) \mu)$ is conditionally gaugeable for all $\varepsilon \in\left[0, \varepsilon_{0}\right]$.

Proof. Note that

$$
\begin{gathered}
\inf \left\{\mathcal{E}(u, u)+\left(1-\varepsilon_{0}\right) \int_{E} u(x)^{2} \mu^{-}(d x): u \in \mathcal{F} \text { with } \int_{E} u(x)^{2}\left(1+\varepsilon_{0}\right) \mu^{+}(d x)=1\right\} \\
\geq \frac{1-\varepsilon_{0}}{1+\varepsilon_{0}} \inf \left\{\mathcal{E}(u, u)+\int_{E} u(x)^{2} \mu^{-}(d x): u \in \mathcal{F} \text { with } \int_{E} u(x)^{2} \mu^{+}(d x)=1\right\}
\end{gathered}
$$

which will be larger than 1 if $\varepsilon_{0} \in(0,1)$ is small enough under the assumption that $(X, \mu)$ is either gaugeable or conditionally gaugeable. Thus $\left(X, \mu+\varepsilon_{0}|\mu|\right)$ is either gaugeable or conditionally gaugeable by Theorems 5.2 and 5.3 . The remaining assertions follow from the observation that $\left(\mu+\varepsilon_{0}|\mu|\right)-(1+\varepsilon) \mu$ is a nonnegative smooth measure when $\varepsilon \in\left[0, \varepsilon_{0}\right]$.

Theorem 5.3 was first proved by Takeda [40] using a different approach, under some additional assumptions on the process $X$ and its Green functions (Assumptions G, P and D there). Analytic characterizations such as those in Theorems 5.2 and 5.3 are very useful in determining whether $(X, \mu)$ is gaugeable or conditionally gaugeable in concrete cases. We refer the reader to 40] for some interesting examples where analytic characterizations are used to give explicit conditions on when $(X, \mu)$ is gaugeable or conditionally gaugeable, where $X$ is a Brownian motion or a symmetric stable process on $\mathbf{R}^{n}$ and $\mu$ is a linear combination of surface measures of concentric spheres.

We also refer readers to Chung and Zhao [13] and Zhao [43] for equivalent conditions for gaugeability and conditional gaugeability in the classical Brownian motion setting, to Chen and Song [7] for equivalent conditions of gaugeability for symmetric stable processes in bounded domains, to Chung and Rao [12] for general gaugeability in a related setting, and the references therein for other related works.

Analytic characterizations for the gaugeability and conditional gaugeability of $\left(X, A^{\mu}+F\right)$ will be addressed in a subsequent paper.

\section{ACKNowledgement}

The author thanks M. Takeda for helpful comments on a preliminary version of this paper. He is grateful to the referee for very helpful comments and suggestions for improvements, especially for providing those remarks which are stated in Proposition 2.4(2) and Remark 2.3.

\section{REFERENCES}

1. M. Aizenman and B. Simon, Brownian motion and Harnack inequality for Schrödinger operators. Comm. Pure Appl. Math. 35 (1982), 209-273. MR 84a:35062

2. A. B. Amor and W. Hansen, Continuity of eigenvalues for Schrödinger operators, $L^{p}$-properties of Kato type integral operators, Math. Ann. 321 (2001), 925-953. 
3. A. Benveniste and J. Jacod, Systèmes de Lévy des processus de Markov. Invent. Math. 21 (1973), 183-198. MR 49:8117

4. R. M. Blumenthal and R. K. Getoor, Markov Processes and Potential Theory. Academic Press, New York, 1968. MR 41:9348

5. Z.-Q. Chen, Z.-M. Ma, and M. Röckner, Quasi-homeomorphisms of Dirichlet forms. Nagoya Math. J. 136 (1994), 1-15. MR 95m:31020

6. Z.-Q. Chen and R. Song, Estimates on Green functions and Poisson kernels of symmetric stable processes. Math. Ann. 312 (1998), 465-601. MR 2000b:60179

7. Z.-Q. Chen and R. Song, Intrinsic ultracontractivity and conditional gauge for symmetric stable processes. J. Funct. Anal. 150 (1997), 204-239. MR 98j:60103

8. Z.-Q. Chen and R. Song, General gauge and conditional gauge theorems. Preprint, 2000. To appear in Ann. Probab.

9. Z.-Q. Chen and R. Song, Conditional gauge theorem for non-local Feynman-Kac transforms. Preprint, 2001. To appear in Probab. Theory Related Fields.

10. Z.-Q. Chen and R. Song, Drift transforms and Green function estimates for discontinuous processes. Preprint, 2001.

11. K. L. Chung and K. M. Rao, Feynman-Kac functional and the Schrödinger equation. In Seminar on stochastic processes, pp. 1-29, Boston, 1981. Birkhäuser. MR 83g:60089

12. K. L. Chung and K. M. Rao, General gauge theorem for multiplicative functionals. Trans. Amer. Math. Soc. 306 (1988), 819-836. MR 89d:60136

13. K. L. Chung and Z. Zhao, From Brownian motion to Schrödinger's Equation. Springer, Berlin, 1995. MR 96f:60140

14. M. Cranston, E. Fabes and Z. Zhao, Conditional gauge and potential theory for the Schrödinger operator. Trans. Amer. Math. Soc., 307 (1988), 174-194. MR 90a:60135

15. C. Dellacherie and P.-A. Meyer, Probabilités et Potentiel, Chapites V à VIII. Hermann, 1980. MR 82b:60001

16. C. Doléans-Dade, Quelques applications de la formule de changement de variables pour les semimartingales. Z. Wahrsch. 16 (1970), 181-194. MR 44:1113

17. P. J. Fitzsimmons, On the excursions of Markov processes in classical duality. Probab. Theory Related Fields, 75 (1987) 159-178. MR 88g:60174

18. P. J. Fitzsimmons, Time changes of symmetric Markov processes and a Feynman-Kac formula. J. Theoret. Probab. 2 (1989), 487-501. MR 91h:60076

19. P. J. Fitzsimmons and R. K. Getoor, Revuz measures and time changes. Math. Z. 199 (1988), 233-256. MR 89h:60124

20. P. J. Fitzsimmons and R. K. Getoor, Smooth measures and continuous additive functionals of right Markov processes. In "Ito's Stochastic Calculus and Probability Theory", N. Ikeda, S. Watanabe, M. Fukushima and H. Kunita (eds.) Springer-Verlag, Tokyo, 1996. MR 98g:60137

21. G. B. Folland, Real Analysis. John Wiley and Sons, Inc. 1984. MR 86k:28001

22. M. Fukushima, Y. Oshima and M. Takeda, Dirichlet forms and symmetric Markov processes. Walter de Gruyter, Berlin, 1994. MR 96f:60126

23. R. K. Getoor, Transience and recurrence of Markov processes. In Séminaire de Probabilités XIV, Lect. Notes Math. 784 (1980), 397-409. MR 82c:60131

24. R. K. Getoor, Measure perturbations of Markov semigroups. Potential Analysis, 11 (1999), 101-133. MR 2001c:60119

25. R. K. Getoor and J. Glover, Riesz decompositions in Markov process theory. Trans. Amer. Math. Soc. 285 (1984), 107-132. MR 86b:60128

26. T. Kulczycki, Properties of Green function of symmetric stable processes. Probab. Math. Stat., 17(2) (1997), 339-364. MR 98m:60119

27. H. Kunita and T. Watanabe, Notes on transformations of Markov processes connected with multiplicative functionals. Mem. Fac. Sci. Kyushu Univ. Ser. A 17 (1963), 181-191. MR 29:661

28. Z.-M. Ma and M. Röckner, Introduction to the Theory of (Non-symmetric) Dirichlet Forms. Springer-Verlag, Berlin, 1992. MR 94d:60119

29. P. A. Meyer, Note sur l'interpretation des mesures d'equilibre. Seminaire de Probabilités VII, Lect. Notes Math. 321 (1973), 210-216. MR 51:9232

30. Y. Pinchover, Criticality and ground states for second-order elliptic equations. J. Differential Equations 80 (1989), 237-250. MR 91c:35046 
31. D. Revuz, Mesures associés aux fonctionelles additives de Markov, I. Trans. Amer. Math. Soc. 148 (1970), 501-531. MR 43:5611

32. S. Sato, An inequality for the spectral radius of Markov processes, Kodai Math. J. 8, (1985) 5-13. MR 86h:60144

33. M. Sharpe, General Theory of Markov Processes, Academic Press, Boston, 1988. MR 89m:60169

34. M. L. Silverstein, The sector condition implies that semipolar sets are quasi-polar. Z. Wahrsch. 41 (1977), 13-33. MR 57:7784

35. B. Simon, Schrödinger semigroups. Bull. Amer. Math. Soc. 7 (1982), 447-526. MR 86b:81001a

36. P. Stollmann and J. Voigt, Perturbation of Dirichlet forms by measures. Potential Anal. 5 (1996), 109-138. MR 97e:47065

37. W. Stummer and K.-Th Sturm, On exponentials of additive functionals. Stochastic Process. Appl. 85 (2000), 45-60. MR 2001b:60093

38. K.-Th Sturm, Gauge theorems for resolvents with application to Markov processes. Probab. Theory Related Fields 89 (1991), 387-406. MR 93d:60119

39. M. Takeda, Exponential decay of lifetimes and a theorem of Kac on total occupation times. Potential Analysis, 11 (1999), 235-247. MR 2000i:60084

40. M. Takeda, Conditional gaugeability and subcriticality of generalized Schrödinger operators. Preprint, 2001. To appear in J. Funct. Anal.

41. J. Ying, Dirichlet forms perturbed by additive functionals of extended Kato class. Osaka J. Math. 34 (1997), 933-952. MR 99e:60173

42. Z. Zhao, A probabilistic principle and generalized Schrödinger perturbation. J. Funct. Anal., 101 (1991), 162-176. MR 93f:60116

43. Z. Zhao, Subcriticality and gaugeability of the Schrödinger operator. Trans. Amer. Math. Soc. 334 (1992), 75-96. MR 93a:81041

Department of Mathematics, University of Washington, Seattle, Washington 98195

E-mail address: zchen@math.washington.edu 